\title{
Dynamic analysis of frames with viscoelastic dampers: a comparison of damper models
}

\author{
R. Lewandowski*, A. Bartkowiak and H. Maciejewski ${ }^{\mathrm{a}}$ \\ Department of Civil Engineering, Poznan University of Technology, ul. Piotrowo 5, 60-965 Poznan, Poland
}

(Received November 12, 2010, Revised July 20, 2011, Accepted December 13, 2011)

\begin{abstract}
Frame structures with viscoelastic (VE) dampers mounted on them are considered in this paper. It is the aim of this paper to compare the dynamic characteristics of frame structures with VE dampers when the dampers are modelled by means of different models. The classical rheological models, the model with the fractional order derivative, and the complex modulus model are used. A relatively large structure with VE dampers is considered in order to make the results of comparison more representative. The formulae for dissipation energy are derived. The finite element method is used to derive the equations of motion of the structure with dampers and such equations are written in terms of both physical and state-space variables. The solution to motion equations in the frequency domain is given and the dynamic properties of the structure with VE dampers are determined as a solution to the appropriately defined eigenvalue problem. Several conclusions concerning the applicability of a family of models of VE dampers are formulated on the basis of results of an extensive numerical analysis.
\end{abstract}

Keywords: dynamics of frames; viscoelastic dampers; dynamic characteristics; classical rheological models; Kelvin model with fractional order derivative; complex modulus model

\section{Introduction}

Viscoelastic (VE) dampers are energy dissipation devices of passive type, frequently used to mitigate excessive vibration of structures due to winds or earthquakes. A number of applications of VE dampers in civil engineering are listed in the book by Christopoulos and Filiatrault (2006). The properties of VE dampers, such as the possibility of energy dissipation and stiffness, are frequency and temperature dependent and are commonly defined in terms of experimentally obtained storage and loss modules. Good understanding of the dynamic behaviour of dampers is required for the analysis of structures supplemented with VE dampers. The frequency dependence of the properties of VE dampers can be described by means of rheological models or using the complex modulus model.

In the past, several rheological models were proposed to describe the dynamic behaviour of VE dampers. Both the classical and the so-called fractional-derivative models of VE dampers are available. These models are presented by Chang and Singh (2009) and by Lewandowski and Chorąźyczewski (2010). Very frequently the classical Maxwell or Kelvin models are used.

\footnotetext{
*Corresponding author, Professor, E-mail: roman.lewandowski@put.poznan.pl
}

${ }^{\text {a }}$ Research Scholar 
Each group of models have their own advantages and disadvantages. The generalized rheological models, like the generalized Kelvin model or the generalized Maxwell model, contain more parameters than the fractional ones but lead to the traditional differential equations of motion instead of to the fractional differential equations when the fractional-derivative model of VE dampers is used, as shown by Chang and Singh (2002). In general, the fractional differential equations are more difficult to solve than the ordinary differential equations. The dynamic characteristic of structures with VE dampers, like the frequencies and modes of vibration and the non-dimensional damping ratios, are obtained as the solution to the linear or quadratic eigenvalue problem when the classical rheological models of VE dampers are used. The fractional-derivative models of dampers require a solution to the nonlinear eigenvalue problem, as proposed by Lewandowski and Pawlak (2010), or to the extremely large linear eigenvalue problem derived, for example, by Chang and Singh (2002) or by Sorrentino and Fassana (2007). The modal strain method is also used to determine the nondimensional damping ratios in the papers by Xu (2007) and by Shen et al. (1995).

The dynamic analysis of frame or building structures with dampers are presented in many papers (Chang and Singh 2002, 2009, Lewandowski and Pawlak 2010, Hatada et al. 2000, Singh and Moreschi 2002, Matsager and Jangid 2005, Singh et al. 2003, Zhang and Xu 2000, Shukla and Datta 1999, Park et al. 2004, Lee et al. 2004, Tsai and Chang 2002, Okada et al. 2006, Mazza and Vulcano (2007)). The simple Maxwell model was chosen by Hatada et al. (2000), Singh and Moreschi (2002) and by Singh et al. (2003). Moreover, in the papers by Singh and Moreschi (2002), Matsager and Jangid (2005) and by Lee et al. (2004) the simple Kelvin model is used to describe the dynamic behaviour of dampers. These simple models are used by Singh and Moreschi (2002), Park et al. (2004) and by Lee et al. (2004), to solve the problem of optimal design of structures with VE dampers. In the papers by Chang and Singh (2002), Lewandowski and Pawlak (2010), and by Tsai and Chang (2002), the three-parameter fractional-derivative rheological model is used to model the dampers behaviour. Moreover, in the paper by Okada et al. (2006), rational polynomial approximation modelling is used for the analysis of structures with VE dampers.

Up to now, the dynamic analysis of structures with VE dampers modelled by means of the generalized rheological models is rarely considered or discussed in the literature. The in-time analysis of structures with dampers modelled by means of the models mentioned above can only be found in the papers by Singh and Chang (2009) and Mazza and Vulcano (2007).

Despite their popularity, the complex modulus model is not used in the context of dynamic analysis of structures with VE dampers.

Design of structures with VE dampers together with optimization of damper locations and parameters are important problems from a practical point of view. Often the concept of performance-based design is adopted in the process of designing of such structures. A detailed review of all the papers is beyond the scope of this paper. Among other ones, we would like to mention the books by Takewaki (2009) and by Christopoulos and Filiatrault (2006) and recent papers by Fujita et al. (2010) and by Mazza and Vulcano (2007). Moreover, the concept of motion-based design is introduced by Connor and Klink (1996).

\section{Motivation, aims and scope of the paper}

The review of existing literature suggests that there are many possible rheological models which are or could be chosen to describe the properties of VE dampers. These models are usually chosen 
intuitively and the choice of the best model is not an obvious one. There is no comparison between the results obtained for different models of dampers. In particular, the effect of VE dampers model on the behaviour of a structure with dampers as a whole is not known. The dynamic characteristics of structure (i.e., natural frequencies, non-dimensional damping ratios, modes of vibration, and frequency response functions) depend on the properties of structure and dampers only while they do not depend on earthquake. These quantities are general and appropriate for the analysis of influence of VE damper models on the overall behaviour of structure. In particular, the non-dimensional damping ratios measure the possibility of dissipation of energy introduced to structures by excitation forces. However, this approach can be used only when structures is elastic.

Of course it is also possible to compare the responses of structures with dampers given in time domain. In this case, we can compare the responses of structures which behave in a nonlinear manner but conclusions which can be drawn from such an analysis are restricted to the earthquakes which are taken into account. The comparison of transient response of simple dynamic systems with different damping models is presented in the paper by Barkanov et al. (2003).

The aim of this paper is to compare the dynamic characteristics of structures with VE dampers when the dampers are modelled by means of different models. A comparison in time domain is beyond the scope of the present paper. A relatively large structure with VE dampers is considered in order to make the results of comparison more representative. Planar frame structures, treated as linear elastic systems, with the VE dampers mounted on the structures are considered. The simple and generalized Kelvin and Maxwell models, the fractional-derivative Kelvin model, and the complex modulus model are used as models of VE dampers.

The outline of the paper is as follows. A description of the rheological models of VE dampers is presented in Section 2. Moreover, formulae for dissipation energy are derived. In Section 3 the equations of motion of the whole system (the structure with dampers) are derived using the finite element method and they are written in terms of both physical and state-space variables. A solution to the motion equations of a whole system in the frequency domain is derived and the dynamic properties of the structure with VE dampers are determined. The frequencies of vibration, the nondimensional damping ratios together with the corresponding eigenvectors are determined as a solution to the appropriately defined eigenvalue problem. The frequency response functions are also determined. In Section 4 a comparison of the effect of damper models on the dynamic properties of structures with VE dampers is made on the basis of results of numerical calculation obtained for a representative frame with VE dampers. Section 5 contains concluding remarks concerning the influence of the damper model on the dynamic characteristics of the structure with VE dampers and suggestions concerning the number of elements of generalized rheological models. Some useful formulae are given in appendices.

\section{Description of models of dampers}

\subsection{Equation of motion of the generalized rheological models}

The frequency dependence of the properties of VE dampers can be captured using generalized rheological models. First of all, the generalized Kelvin model and the generalized Maxwell model are considered. The generalized Kelvin model is built from the spring and a set of the Kelvin elements connected in series (see Fig. 1). The serially connected spring and dashpot will be referred 


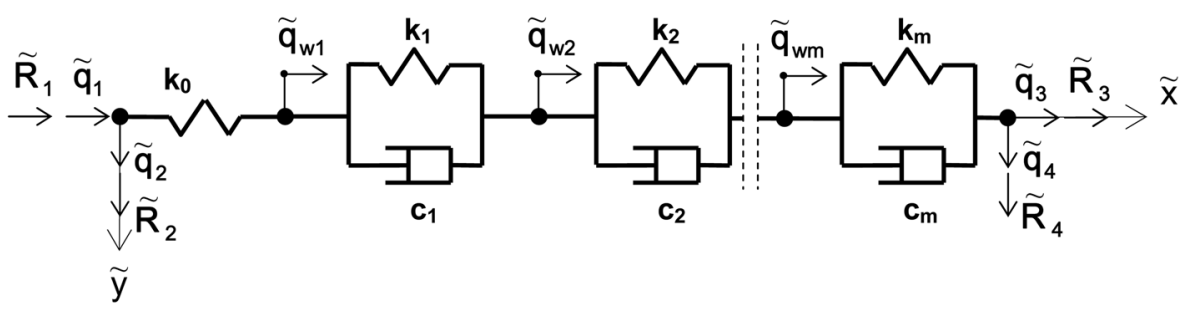

Fig. 1 Scheme of the generalized Kelvin model

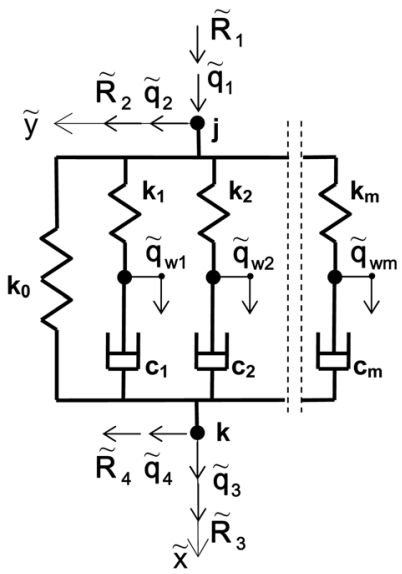

Fig. 2 Scheme of the generalized Maxwell model

to as the Kelvin element. The generalized Maxwell model is built from the spring and a set of the Maxwell elements connected in parallel (see Fig. 2). The Maxwell element is the classical Maxwell rheological model, i.e., the spring and dashpot connected in series. The number of the Kelvin and the Maxwell elements is denoted by $m$.

Using the concept of internal variables, the dynamic behaviour of the Kelvin damper can be described by means of the following equations

$$
\begin{gathered}
u_{0}(t)=k_{0}\left(\tilde{q}_{w, 1}(t)-\tilde{q}_{1}(t)\right) \\
u_{i}(t)=k_{i}\left(\tilde{q}_{w, i+1}(t)-\tilde{q}_{w, i}(t)\right)+c_{i}\left(\dot{\tilde{q}}_{w, i+1}(t)-\dot{\tilde{q}}_{w, m}(t)\right) \\
u_{m}(t)=k_{m}\left(\tilde{q}_{3}(t)-\tilde{q}_{w, m}\right)+c_{m}\left(\dot{\tilde{q}}_{3}(t)-\dot{\tilde{q}}_{w, m}\right)
\end{gathered}
$$

where $u_{i}(t)$ is the force in the $i$-th element of the model $(i=0,1, \ldots, m), k_{i}$ and $c_{i}$ are the spring stiffness and the damping factor of the dashpot of the $i$-th element of model, respectively, and symbols $\tilde{q}_{1}(t)$ and $\tilde{q}_{3}(t)$ denote the external nodes displacements given in the local coordinate system (compare Fig. 1). Moreover, the dot denotes differentiation with respect to time $t$ and the symbol $\tilde{q}_{w, i}(t)$ denotes the internal variable $(i=1, \ldots, m)$.

After introducing the vector of external reactions $\tilde{\mathbf{R}}_{z}(t)=\operatorname{col}\left(\tilde{R}_{1}(t), \tilde{R}_{2}(t), \tilde{R}_{3}(t), \tilde{R}_{4}(t)\right.$ ) (see Fig. 1) and utilizing the equilibrium conditions of the external nodes: $R_{1}(t)=-u_{0}(t), R_{2}(t)=0$, $R_{1}(t)=u_{m}(t)$ and $R_{4}(t)=0$ we can write the following matrix equation 


$$
\tilde{\mathbf{R}}_{z}(t)=\tilde{\mathbf{K}}_{z z} \tilde{\mathbf{q}}_{z}(t)+\tilde{\mathbf{K}}_{z w} \tilde{\mathbf{q}}_{w}(t)+\tilde{\mathbf{C}}_{z z} \dot{\tilde{\mathbf{q}}}_{z}(t)+\tilde{\mathbf{C}}_{z w} \dot{\tilde{\mathbf{q}}}_{w}(t)
$$

where $\tilde{\mathbf{q}}_{z}(t)=\operatorname{col}\left(\tilde{q}_{1}(t), \tilde{q}_{2}(t), \tilde{q}_{3}(t), \tilde{q}_{4}(t)\right), \tilde{\mathbf{q}}_{w}(t)=\operatorname{col}\left(\tilde{q}_{w, 1}(t), \ldots, \tilde{q}_{w, m}(t)\right)$.

Moreover, the equilibrium conditions of the internal nodes i.e., $u_{i-1}(t)-u_{1}(t)=0$ for $i=1, \ldots, m$ lead to the following matrix equation

$$
\tilde{\mathbf{K}}_{w z} \tilde{\mathbf{q}}_{z}(t)+\tilde{\mathbf{K}}_{w w} \tilde{\mathbf{q}}_{w}(t)+\tilde{\mathbf{C}}_{w z} \dot{\tilde{\mathbf{q}}}_{z}(t)+\tilde{\mathbf{C}}_{w w} \dot{\tilde{\mathbf{q}}}_{w}(t)=0
$$

where $\tilde{\mathbf{K}}_{w z}=\tilde{\mathbf{K}}_{z w}^{T}, \tilde{\mathbf{C}}_{w z}=\tilde{\mathbf{C}}_{z w}^{T}$.

The VE damper Kelvin model equation written in the local coordinate system can be finally presented in the form

$$
\tilde{\mathbf{R}}_{d}(t)=\tilde{\mathbf{K}}_{d} \tilde{\mathbf{q}}_{d}(t)+\tilde{\mathbf{C}}_{d} \dot{\tilde{\mathbf{q}}}_{d}(t)
$$

where $\tilde{\mathbf{R}}_{d}(t)=\operatorname{col}\left(\tilde{\mathbf{R}}_{z}(t), \mathbf{0}\right), \tilde{\mathbf{q}}_{d}(t)=\operatorname{col}\left(\tilde{\boldsymbol{q}}_{z}(t), \tilde{\mathbf{q}}_{w}(t)\right)$

$$
\tilde{\mathbf{K}}_{d}=\left[\begin{array}{cc}
\tilde{\mathbf{K}}_{z z} & \tilde{\mathbf{K}}_{z w} \\
\tilde{\mathbf{K}}_{w z} & \tilde{\mathbf{K}}_{w w}
\end{array}\right], \quad \tilde{\mathbf{C}}_{d}=\left[\begin{array}{ll}
\tilde{\mathbf{C}}_{z z} & \tilde{\mathbf{C}}_{z w} \\
\tilde{\mathbf{C}}_{w z} & \tilde{\mathbf{C}}_{w w}
\end{array}\right]
$$

In this paper, the unusual transformation of nodal parameters to the global coordinate system is used. The displacements of the damper external nodes are transformed as usually but the internal variables of damper are still defined in the local coordinate system. It means that the transformation matrix is

$$
\mathbf{T}_{d}=\left[\begin{array}{cc}
\tilde{\mathbf{T}}_{d} & \mathbf{0} \\
\mathbf{0} & \mathbf{I}
\end{array}\right]
$$

where

$$
\tilde{\mathbf{T}}_{d}=\left[\begin{array}{cc}
\tilde{\mathbf{T}} & \mathbf{0} \\
\mathbf{0} & \tilde{\mathbf{T}}
\end{array}\right], \tilde{\mathbf{T}}=\left[\begin{array}{cc}
c & s \\
-s & c
\end{array}\right]
$$

$c=\cos \alpha, s=\sin \alpha, \alpha$ is the angle between the global and local coordinate system and $\mathbf{I}$ is the $(m \times m)$ identity matrix.

The generalized Kelvin model, which is a set of elements connected together by hinges, is a geometrically movable system. The stiffness matrix of such a model system written in the global coordinate system and using the usual transformation matrix is singular and the global stiffness matrix of a structure with dampers is also singular. The above-mentioned unusual transformation guarantees that the global matrix of the system is not singular.

After transformation of Eq. (7) to the global coordinate system we obtain

$$
\mathbf{R}_{d}(t)=\mathbf{K}_{d} \mathbf{q}_{d}(t)+\mathbf{C}_{d} \dot{\mathbf{q}}_{d}(t)
$$

where $\mathbf{R}_{d}(t)=\operatorname{col}\left(\mathbf{R}_{z}(t), \mathbf{0}\right)=\mathbf{T}_{d}^{T} \tilde{\mathbf{R}}_{d} \mathbf{T}_{d}, \mathbf{R}_{z}(t)=\operatorname{col}\left(R_{1}(t), R_{2}(t), R_{3}(t), R_{4}(t)\right), \mathbf{q}_{d}(t)=\operatorname{col}\left(\mathbf{q}_{z}(t)\right.$, $\left.\mathbf{q}_{w}(t)=\tilde{\mathbf{q}}_{w}(t)\right)=\mathbf{T}_{d}^{T} \tilde{\mathbf{q}} \mathbf{T}_{d}, \mathbf{q}_{z}(t)=\operatorname{col}\left(q_{1}(t), q_{2}(t), q_{3}(t), q_{4}(t)\right)$, are the vector of nodal reactions and the vector of nodal parameters, respectively in the global coordinate system. The explicit forms of matrices $\mathbf{K}_{d}$ and $\mathbf{C}_{d}$ are given in Appendix A.

The dynamic behaviour of the Maxwell damper could be described in a similar way. In terms of 
nodal displacements and internal variables, as defined in Fig. 2, the following equations could be written

$$
u_{0}(t)=k_{0}\left(\tilde{q}_{3}(t)-\tilde{q}_{1}(t)\right), u_{i s}(t)=k_{i}\left(\tilde{q}_{w, i}(t)-\tilde{q}_{1}(t)\right), u_{i d}(t)=c_{i}\left(\dot{\tilde{q}}_{3}(t)-\dot{\tilde{q}}_{w, i}(t)\right)
$$

for the spring element and for the $i$-th Maxwell element $(i=1, \ldots, m)$. The symbols $u_{0}(t), u_{i s}(t)$ and $u_{i d}(t)$ denote the force in the spring element, the force in the spring of the $i$-th Maxwell element, and the force in the dashpot of the $i$-th Maxwell element, respectively.

The nodal reactions in the local coordinate system are

$$
\tilde{R}_{1}(t)=-u_{0}(t)-\sum_{i=1}^{m} u_{i s}(t), \tilde{R}_{2}(t)=0, \tilde{R}_{3}(t)=u_{0}(t)+\sum_{i=1}^{m} u_{i d}(t), \tilde{R}_{4}(t)=0
$$

After introducing relationships (12) into Eq. (13) we obtain again the matrix Eq. (4). In the global coordinate system, Eq. (11) is also valid with the matrices $\mathbf{K}_{d}$ and $\mathbf{C}_{d}$ given in Appendix B.

Many particular rheological models existing in the literature can be obtained by varying the number of elements in the generalized models mentioned above.

\subsection{Equation of motion of the simple rheological models, the fractional-derivative Kelvin model, and the complex modulus model}

The simple Kelvin and Maxwell models, which contain only one Kelvin or Maxwell element, respectively, are not particular instances of the discussed generalized models because the spring element with stiffness $k_{0}$ is not present. The simple Kelvin model and the simple Maxwell model are shown in Fig. 3.

The matrix Eqs. (7) and (11) are equations of simple models treated as the finite elements. The matrices $\mathbf{K}_{d}$ and $\mathbf{C}_{d}$ are given in Appendix $\mathbf{C}$.

The fractional-derivative Kelvin model is shown in Fig. 4. The equation of motion of the model can be written in the following form

$$
u(t)=k_{1}\left(\tilde{q}_{3}(t)-\tilde{q}_{1}(t)\right)+c_{1} D_{t}^{\alpha}\left(\tilde{q}_{3}(t)-\tilde{q}_{1}(t)\right)
$$

where the symbol $D_{t}^{\alpha}(\bullet)$ denotes the Riemann-Liouville fractional-derivative of the order $\alpha$ $(0<\alpha \leq 1)$ with respect to time, $t$. For additional information concerning the Riemann-Liouville fractional-derivative, Podlubny (1999) may be consulted.

The matrix equation of the fractional-derivative Kelvin damper written in the local coordinate system could be written in the form

$$
\tilde{\mathbf{R}}_{d}(t)=\tilde{\mathbf{K}}_{d} \tilde{\mathbf{q}}_{d}(t)+\tilde{\mathbf{C}}_{d} D_{t}^{\alpha} \tilde{\mathbf{q}}_{d}(t)
$$

(a)

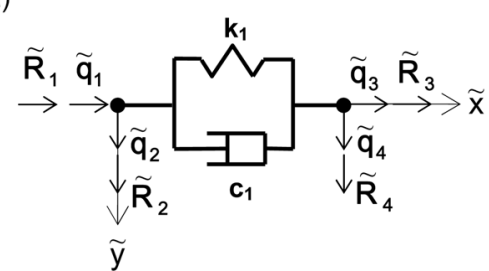

(b)

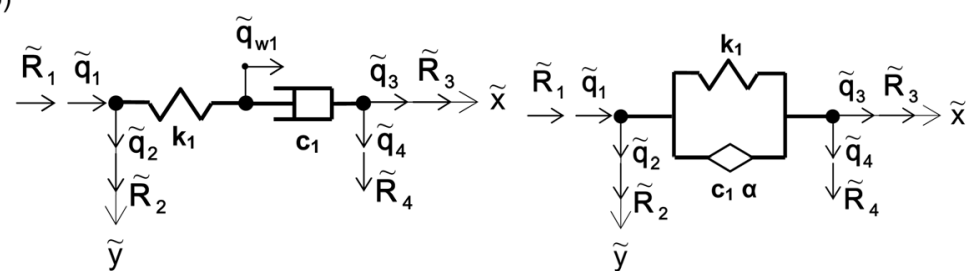

Fig. 3 Scheme of (a) the simple Kelvin model and (b) the simple Maxwell Fig. 4 Scheme of the fractional model derivative Kelvin model 
In the global coordinate system we can write the following equation

$$
\mathbf{R}_{d}(t)=\mathbf{K}_{d} \mathbf{q}_{d}(t)+\mathbf{C}_{d} D_{t}^{\alpha} \mathbf{q}_{d}(t)
$$

where, again, matrices $\mathbf{K}_{d}$ and $\mathbf{C}_{d}$ are given in Appendix C.

The equation of motion of dampers modelled using the complex modulus model is

$$
u(t)=K\left(\tilde{q}_{3}(t)-\tilde{q}_{1}(t)\right)
$$

where $K$ is now the damper dynamic stiffness.

For the damper executing the harmonic motion, i.e., when $u(t)=u_{0} \exp (\mathrm{i} \lambda t), \tilde{q}_{1}(t)=\tilde{q}_{10} \exp (\mathrm{i} \lambda t)$, $\tilde{q}_{3}(t)=\tilde{q}_{30} \exp (\mathrm{i} \lambda t)$ the following equation could be written

$$
u_{0}=\left(K^{\prime}(\lambda)+i K^{\prime \prime}(\lambda)\right)\left(\tilde{q}_{30}-\tilde{q}_{10}\right)
$$

in the frequency domain. Above $i=\sqrt{-1}$ is the imaginary unit, $K^{\prime}(\lambda)$ and $K^{\prime \prime}(\lambda)$ are the storage modulus and the loss modulus, respectively.

The finite element equations of this model written in the frequency domain and in the local and global coordinate systems are

$$
\tilde{\mathbf{R}}_{d 0}=\left(\tilde{\mathbf{K}}_{d}^{\prime}(\lambda)+\tilde{\mathbf{K}}_{d}^{\prime \prime}(\lambda)\right) \tilde{\mathbf{q}}_{d 0}, \mathbf{R}_{d 0}=\left(\mathbf{K}_{d}^{\prime}(\lambda)+\mathbf{K}_{d}^{\prime \prime}(\lambda)\right) \mathbf{q}_{d 0}
$$

respectively, where matrices $\mathbf{K}_{d}^{\prime}(\lambda) \mathbf{K}_{d}^{\prime \prime}(t)$ are given in Appendix C. In Eq. (19) the symbols $\tilde{\mathbf{R}}_{d 0}(t)=\operatorname{col}\left(\tilde{R}_{10}\left((t), \tilde{R}_{20}(t), \tilde{R}_{30}(t), \tilde{R}_{40}(t)\right)\right), \mathbf{R}_{d 0}(t)=\operatorname{col}\left(R_{10}(t), R_{20}(t), R_{30}(t), R_{40}(t)\right), \tilde{\mathbf{q}}_{d 0}(t)=$ $\operatorname{col}\left(\tilde{q}_{10}(t), \tilde{q}_{20}(t), \tilde{q}_{30}(t), \tilde{q}_{40}(t)\right)$ and $\mathbf{q}_{d 0}(t)=\operatorname{col}\left(q_{10}(t), q_{20}(t), q_{30}(t), q_{40}(t)\right)$ denote the vector of complex amplitudes of reaction and nodal displacements in the local and global coordinate systems, respectively.

The above-mentioned storage and loss modulus are important characteristics of VE dampers. For all of the considered models of dampers the appropriate formulae are collected in Appendix D.

\subsection{Energy dissipated in the harmonically excited damper}

The energy dissipated by the damper is an important characteristic of the VE damper. Usually the energy dissipated by a damper making harmonic motions is used as a measure of this property. To determine such energy, for the generalized Kelvin damper, it is assumed that the left external node of damper is fixed while the right node is harmonically excited, i.e.

$$
q_{1}(t)=q_{2}(t)=q_{4}(t)=0, q_{3}(t)=a_{3} \sin \lambda t+b_{3} \cos \lambda t
$$

where $\lambda$ is the excitation frequency, symbols $a_{3}$ and $b_{3}$ denote the known amplitudes of harmonic excitation. In this subsection, the equation of motion in the local coordinate system is used but waves over the symbols are omitted to simplify the notation.

In the considered case, the dynamic behaviour of damper is described by

$$
\mathbf{K}_{w w} \mathbf{q}_{w}(t)+\mathbf{C}_{w w} \dot{\mathbf{q}}_{w}(t)=\mathbf{f}_{d}(t)
$$

where $\mathbf{f}_{d}(t)=\operatorname{col}\left(k_{0} q_{1}(t), 0, \ldots, 0, k_{m} q_{3}(t)+c_{m} \dot{q}_{3}(t)\right)$.

The damper steady state motion is harmonic, i.e.

$$
\mathbf{q}_{w}(t)=\mathbf{a} \sin \lambda t+\mathbf{b} \cos \lambda t
$$

where $\mathbf{a}=\operatorname{col}\left(a_{w, 1}, \ldots, a_{w, m}\right)$ and $\mathbf{b}=\operatorname{col}\left(b_{w, 1}, \ldots, b_{w, m}\right)$ are the unknown vectors which could be 
determined from the following set of algebraic equations

$$
\mathbf{K}_{w w} \mathbf{a}-\lambda \mathbf{C}_{w w} \mathbf{b}=\mathbf{f}_{s}, \lambda \mathbf{C}_{w w} \mathbf{a}+\mathbf{K}_{w w} \mathbf{b}=\mathbf{f}_{c}
$$

where $\mathbf{f}_{s}=\operatorname{col}\left(0,0, \ldots, 0, k_{m} a_{3}-\lambda c_{m} b_{3}\right)$ and $\mathbf{f}_{s}=\operatorname{col}\left(0,0, \ldots, 0, k_{m} b_{3}+\lambda c_{m} a_{3}\right)$.

The energy dissipated by the damper can be calculated from the following formula

$$
E_{d}=\int_{0}^{T} \mathbf{u}^{T}(t) \dot{\mathbf{x}}(t) d t
$$

where $T=2 \pi / \lambda$ is the period of excitation, $\mathbf{u}(t)=\operatorname{col}\left(u_{1}(t), u_{2}(t), \ldots, u_{m}(t)\right)$ is the vector of forces in the Kelvin elements and $\dot{\mathbf{x}}(t)=\operatorname{col}\left(\dot{q}_{w, 2}(t)-\dot{q}_{w, 1}(t), \ldots, \dot{q}_{3}(t)-\dot{q}_{w, m}(t)\right)$ is the vector of relative velocities of the Kelvin elements.

Taking into account relationships (1) - (3) and (22) and making the necessary integration the following formula

$$
E_{d}=\pi \lambda^{2}\left(\Delta \mathbf{a}^{T} \mathbf{C}_{d} \Delta \mathbf{a}+\Delta \mathbf{b}^{T} \mathbf{C}_{d} \Delta \mathbf{b}\right)
$$

is finally obtained, where $\mathbf{C}_{d}=\operatorname{diag}\left(c_{1}, \ldots, c_{m}\right), \quad \Delta \mathbf{a}=\operatorname{col}\left(a_{w, 2}-a_{w, 1}, \ldots, a_{3}-a_{w, m}\right), \quad \Delta \mathbf{b}=\operatorname{col}$ $\left(b_{w, 2}-b_{w, 1}, \ldots, b_{3}-b_{w, m}\right)$

The energy dissipated by the Maxwell damper can be obtained in a similar way. As previously, it is assumed that the motion of the left and the right external nodes is described by relationship (20). In this case, the motion of internal variables can be determined irrespective of each other. The motion of the $i$-th Maxwell element is described by the equation

$$
q_{w, i}(t)+\tau_{i} q_{w, i}(t)=q_{1}(t)+\tau_{i} \dot{q}_{3}(t)
$$

The damper steady state vibrations are also described by Eq. (22). After introducing Eq. (22) into Eq. (26) the following result is obtained $(i=1, \ldots, m)$

$$
a_{w, i}=\frac{\left(\tau_{i} \lambda\right)^{2} a_{3}-\tau_{i} \lambda b_{3}}{1+\left(\tau_{i} \lambda\right)^{2}}, b_{w, i}=\frac{\left(\tau_{i} \lambda\right)^{2} b_{3}+\tau_{i} \lambda a_{3}}{1+\left(\tau_{i} \lambda\right)^{2}}
$$

The dissipation energy is also calculated from relationship (24) from which the final formula (25) is obtained but the definitions of vectors $\Delta \mathbf{a}$ and $\Delta \mathbf{b}$ are slightly different, i.e.

$$
\Delta \mathbf{a}=\operatorname{col}\left(a_{3}-a_{w, 1}, \ldots, a_{3}-a_{w, m}\right), \Delta \mathbf{b}=\operatorname{col}\left(b_{3}-b_{w, 1}, \ldots, b_{3}-b_{w, m}\right)
$$

The energy dissipated by the simple Kelvin damper and by the simple Maxwell damper can be calculated in a similar way. The final formulae are given in Appendix C.

Some explanation is needed for the fractional-derivative Kelvin model. In this case, $x(t)=q_{3}(t)-q_{1}(t)$, the relationships (20) are also valid and after introducing (20) into (14) and taking into account that (compare Podlubny (1999))

$$
D_{t}^{\alpha} \sin \lambda t=\lambda^{\alpha} \sin (\lambda t+\alpha \pi / 2), D_{t}^{\alpha} \cos \lambda t=\lambda^{\alpha} \cos (\lambda t+\alpha \pi / 2)
$$

From (24) it is obtained the following final result

$$
E_{d}=\pi \lambda c\left(a_{b}^{2}+b_{3}^{2}\right) \sin (\alpha \pi / 2)
$$

The energy dissipated by the damper, modeled using the complex modulus model is

$$
E_{d}=\pi K^{\prime \prime}(\lambda) q_{30}^{2}
$$




\section{Determination of dynamic characteristic of structures with VE dampers}

The considered frame structures with VE dampers are modeled using the finite element method. The structure is treated as the linear system. The typical two node bar element with the sixth nodal parameters is used to describe the structure. The mass and stiffness matrices together with the vector of nodal forces of the element can be found in many books. The equation of motion of the structure with VE dampers modeled using the generalized rheological models can be written in the form

$$
\begin{gathered}
\mathbf{M}_{s s} \ddot{\mathbf{q}}_{s}(t)+\mathbf{C}_{s s} \dot{\mathbf{q}}_{s}(t)+\mathbf{C}_{s d} \dot{\mathbf{q}}_{d}(t)+\mathbf{K}_{s s} \mathbf{q}_{s}(t)+\mathbf{K}_{s d} \mathbf{q}_{d}(t)=\mathbf{p}_{s}(t) \\
\mathbf{C}_{d s} \mathbf{q}(t)+\mathbf{C}_{d d} \dot{\mathbf{q}}_{d}(t)+\mathbf{K}_{d s} \mathbf{q}_{s}(t)+\mathbf{K}_{d d} \mathbf{q}_{d}(t)=0
\end{gathered}
$$

where symbols $\mathbf{M}_{s s}, \mathbf{C}_{s s}, \mathbf{C}_{s d}=\mathbf{C}_{d s}^{T}, \mathbf{C}_{d d}, \mathbf{K}_{s s}, \mathbf{K}_{s d}=\mathbf{K}_{d s}^{T}$ and $\mathbf{K}_{d d}$ denote the mass, damping and stiffness matrices of the structure with dampers, respectively, written in the global coordinate system. The dimension of matrices $\mathbf{M}_{s s}, \mathbf{C}_{s s}=\mathbf{C}_{s s}^{(s)}+\mathbf{C}_{s s}^{(d)}$ and $\mathbf{K}_{s s}=\mathbf{K}_{s s}^{(s)}+\mathbf{K}_{s s}^{(d)}$ is $(n \times n)$. Matrices $\mathbf{M}_{s s}, \mathbf{C}_{s s}^{(s)}$ and $\mathbf{K}_{s s}^{(s)}$ describe the inertia, damping and elastic properties of the structure without dampers, while the matrices $\mathbf{C}_{s s}^{(d)}, \mathbf{K}_{s s}^{(d)}$ and the $(n \times r)$ matrices $\mathbf{C}_{s d}=\mathbf{C}_{d s}^{T}, \mathbf{K}_{s d}=\mathbf{K}_{d s}^{T}$ represent the effect of the coupling of dampers with the structure. The $(r \times r)$ matrices $\mathbf{C}_{d d}$ and $\mathbf{K}_{d d}$ describe the damping and stiffness properties of dampers with braces, respectively. Moreover, $\mathbf{q}_{s}(t), \mathbf{q}_{d}(t)$ and $\mathbf{P}_{s}(t)$ are the global vectors of nodal generalized displacements, internal variables and nodal excitation forces, respectively. The concept of proportional damping is used to model the damping properties of the structure, i.e.: $\mathbf{C}_{s s}^{(s)}=\alpha \mathbf{M}_{s s}+\kappa \mathbf{K}_{s s}^{(s)}$ where $\alpha$ and $\kappa$ are proportionality factors.

The equation of motion written in terms of state variables will also be useful. After introducing the following state vector $\mathbf{x}(t)=\operatorname{col}\left(\mathbf{q}_{s}(t), \dot{\mathbf{q}}_{s}(t), \mathbf{q}_{d}(t)\right)$ and adding the equation

$$
\mathbf{M}_{s s} \dot{\mathbf{q}}_{s}(t)-\mathbf{M}_{s s} \dot{\mathbf{q}}_{s}(t)=0
$$

to a system of Eqs. (32) and (33) the following state equation could be written

$$
\mathbf{A} \dot{\mathbf{x}}(t)+\mathbf{B} \mathbf{x}(t)=\mathbf{s}(t)
$$

where

$$
\mathbf{A}=\left[\begin{array}{ccc}
\mathbf{C}_{s s} & \mathbf{M}_{s s} & \mathbf{C}_{s d} \\
\mathbf{M}_{s s} & \mathbf{0} & \mathbf{0} \\
\mathbf{C}_{d s} & \mathbf{0} & \mathbf{C}_{d d}
\end{array}\right], \mathbf{B}=\left[\begin{array}{ccc}
\mathbf{K}_{s s} & \mathbf{0} & \mathbf{K}_{s d} \\
\mathbf{0} & -\mathbf{M}_{s s} & \mathbf{0} \\
\mathbf{K}_{d s} & \mathbf{0} & \mathbf{K}_{d d}
\end{array}\right], \mathbf{s}(t)=\left\{\begin{array}{c}
\mathbf{p}(t) \\
\mathbf{0} \\
\mathbf{0}
\end{array}\right\}
$$

Please note that the matrices $\mathbf{A}$ and $\mathbf{B}$ are symmetric and the matrix $\mathbf{B}$ is non-singular.

The solution to the homogenous state equation, i.e., when $\mathbf{s}(t)=\mathbf{0}$, is assumed in the form

$$
\mathbf{x}(t)=\mathbf{a} \exp (s t)
$$

The linear eigenvalue problem

$$
(s \mathbf{A}+\mathbf{B}) \mathbf{a}=\mathbf{0}
$$

must be solved to determine the $(2 n+r)$ eigenvalues $s_{i}$ and eigenvectors $\mathbf{a}_{i}$. In the case of an undercritically damped structure the $2 n$ eigenvalues (eigenvectors) are the complex and conjugate numbers (vectors) while the remaining $r$ eigenvalues (eigenvectors) are the real numbers (vectors).

The dynamic behavior of a frame with VE dampers is characterized by the natural frequencies $\omega_{i}$ 
and the non-dimensional damping parameters $\gamma_{i}$. The above-mentioned quantities are defined as

$$
\omega_{i}^{2}=\mu_{i}^{2}+\eta_{i}^{2}, \gamma_{i}=-\mu_{i} / \omega_{i}
$$

where $\mu_{i}=\operatorname{Re}\left(s_{i}\right), \eta_{i}=\operatorname{Im}\left(s_{i}\right)$. The formulae (39) refer to complex eigenvalues only.

The third dynamic characteristic of the considered system are the frequency response functions. To determine these functions the steady state harmonic responses of the system are considered. If the excitation forces vary harmonically in time, i.e., when

$$
\mathbf{p}(t)=\mathbf{P} \exp (\mathrm{i} \lambda t)
$$

then the steady state response of the structure and the vector of state variables can be expressed as

$$
\mathbf{q}_{s}(t)=Q_{s} \exp (\mathrm{i} \lambda t), \mathbf{q}_{d}(t)=\mathbf{Q}_{d} \exp (\mathrm{i} \lambda t)
$$

After substituting relationships (40) and (41) into Eqs. (32) and (33), which can be rewritten in the form

$$
\mathbf{M} \ddot{\mathbf{q}}(t)=\mathbf{C} \dot{\mathbf{q}}(t)+\mathbf{K q}(t)=\overline{\mathbf{p}}(t)
$$

where

$$
\mathbf{M}=\left[\begin{array}{cc}
\mathbf{M}_{s s} & \mathbf{0} \\
\mathbf{0} & \mathbf{0}
\end{array}\right], \mathbf{C}=\left[\begin{array}{cc}
\mathbf{C}_{s s} \mathbf{C}_{s d} \\
\mathbf{C}_{d s} \mathbf{C}_{d d}
\end{array}\right], \mathbf{K}=\left[\begin{array}{l}
\mathbf{K}_{s s} \mathbf{K}_{s d} \\
\mathbf{K}_{d s} \mathbf{K}_{d d}
\end{array}\right], \mathbf{q}(t)=\left\{\begin{array}{c}
\mathbf{q}_{s}(t) \\
\mathbf{q}_{d}(t)
\end{array}\right\}, \overline{\mathbf{p}}(t)=\left\{\begin{array}{c}
\mathbf{p}(t) \\
\mathbf{0}
\end{array}\right\}
$$

the following relationship describes the matrix of the frequency response functions

$$
\mathbf{H}(\lambda)=\left(-\lambda^{2} \mathbf{M}+i \lambda \mathbf{C}+\mathbf{K}\right)^{-1}
$$

If the structure with dampers modeled by the simple Maxwell model is considered, then all of the relationships presented above in this section are valid provided that the matrices given in Appendix $\mathrm{C}$ are used to generate the global matrices appearing in Eqs. (32) and (33).

The vector of internal variables $\mathbf{q}_{d}(t)$ and the equation of motion (33) are not present in the case of the structure with dampers modeled by the simple Kelvin model, and the motion Eq. (32) takes the form

$$
\mathbf{M}_{s s} \ddot{\mathbf{q}}_{s}(t)+\left(\mathbf{C}_{s s}+\mathbf{C}_{d d}\right) \dot{\mathbf{q}}_{s}(t)+\left(\mathbf{K}_{s s}+\mathbf{K}_{d d}\right) \mathbf{q}_{s}(t)=\mathbf{p}_{s}(t)
$$

The matrices $\mathbf{C}_{d d}$ and $\mathbf{K}_{d d}$ appearing in (45) are built from the matrices $\mathbf{C}_{d}$ and $\mathbf{K}_{d}$, respectively, given by formulae (C.2).

The state equation has the form of Eq. (35) where now

$$
\mathbf{x}(t)=\left\{\begin{array}{c}
\mathbf{q}_{s}(t) \\
\dot{\mathbf{q}}_{s}(t)
\end{array}\right\}, \mathbf{A}=\left[\begin{array}{cc}
\mathbf{C}_{s s}+\mathbf{C}_{d d} & \boldsymbol{M}_{s s} \\
\mathbf{M}_{s s} & \mathbf{0}
\end{array}\right], \mathbf{B}=\left[\begin{array}{cc}
\mathbf{K}_{s s}+\mathbf{K}_{d d} & 0 \\
\mathbf{0} & -\mathbf{M}_{s s}
\end{array}\right], \mathbf{s}(t)=\left\{\begin{array}{c}
\mathbf{p}(t) \\
0
\end{array}\right\}
$$

Moreover, the matrix of frequency response functions is defined as

$$
\mathbf{H}(\lambda)=\left[-\lambda^{2} \mathbf{M}_{s s}+\mathrm{i} \lambda\left(\mathbf{C}_{s s}+\mathbf{C}_{d d}\right)+\mathbf{K}_{s s}+\mathbf{K}_{d d}\right]^{-1}
$$

In the case of structure with dampers modeled using the fractional-derivative Kelvin model the equation of motion has the form (see paper by Lewandowski and Pawlak 2010)

$$
\mathbf{M}_{s s} D_{t}^{2} \mathbf{q}_{s}(t)+\mathbf{C}_{s s} D_{t}^{1} \mathbf{q}_{s}(t)+\mathbf{C}_{d d} D_{t}^{\alpha} \mathbf{q}_{s}(t)+\left(\mathbf{K}_{s s}+\mathbf{K}_{d d}\right) \mathbf{q}_{s}(t)=\mathbf{p}_{s}(t)
$$


where symbols $D_{t}^{2} \mathbf{q}_{s}(t)=\ddot{\mathbf{q}}_{s}(t)$ and $D_{t}^{1} \mathbf{q}_{s}(t)=\dot{\mathbf{q}}_{s}(t)$ are used in order to be consistent with the notation. Here, it is assumed that for all dampers the values of parameter $\alpha$ are identical.

The state equation is

$$
\mathbf{A} D_{t}^{1} \mathbf{z}(t)+\mathbf{A}_{1} D_{t}^{\alpha} \mathbf{z}(t)+\mathbf{B z}(t)=\mathbf{s}(t)
$$

where $\mathbf{z}(t)=\operatorname{col}\left(\mathbf{q}_{s}(t), D_{t}^{1} \mathbf{q}_{s}(t)\right), \mathbf{s}(t)=\operatorname{col}\left(\mathbf{p}_{s}(t), \mathbf{0}\right)$

$$
\mathbf{A}=\left[\begin{array}{cc}
\mathbf{C}_{s s} & \mathbf{M}_{s s} \\
\mathbf{M}_{s s} & \mathbf{0}
\end{array}\right], \mathbf{A}_{1}=\left[\begin{array}{cc}
\mathbf{C}_{d d} & \mathbf{0} \\
\mathbf{0} & \mathbf{0}
\end{array}\right], \mathbf{B}=\left[\begin{array}{cc}
\mathbf{K}_{s s}+\mathbf{K}_{d d} & \mathbf{0} \\
0 & -\mathbf{M}_{s s}
\end{array}\right]
$$

The eigenvalue problem which must be solved to determine the eigenvalue $s$ and the eigenvector $\mathbf{a}$ is the nonlinear one and is in the following form

$$
\left(s \mathbf{A}+s^{\alpha} \mathbf{A}_{1}+\mathbf{B}\right) \mathbf{a}=0
$$

The above nonlinear eigenvalue problem can be solved using the continuation method described by Lewandowski and Pawlak (2010) and the relationships (39) are used to determine the natural frequencies and non-dimensional damping ratios.

After applying the Laplace transformation on Eq. (48) and assuming zero initial conditions and $\mathbf{p}_{s}(t)=\mathbf{0}$, we obtain the following eigenvalue problem, alternative to (51)

$$
\left(s^{2} \mathbf{M}_{s s}+s \mathbf{C}_{s s}+s^{\alpha} \mathbf{C}_{d d}+\mathbf{K}_{s s}+\mathbf{K}_{d d}\right) \mathbf{a}=\mathbf{0}
$$

The matrix of frequency response functions is defined as

$$
\mathbf{H}(\lambda)=\left[-\lambda^{2} \mathbf{M}_{s s}+i \lambda \mathbf{C}_{s s}+(i \lambda)^{\alpha} \mathbf{C}_{d d}+\mathbf{K}_{s s}+\mathbf{K}_{d d}\right]^{-1}
$$

The equation of motion of structure with VE dampers modeled using the complex modulus model can be written in the form

$$
\mathbf{M}_{s s} \ddot{\boldsymbol{q}}_{s}(t)+\left(\mathbf{K}_{s s}+\overline{\mathbf{K}}_{d d}\right) \mathbf{q}_{s}(t)=\mathbf{p}_{s}(t)
$$

where $\overline{\mathbf{K}}_{d d}$ is the global, complex stiffness matrix of dampers.

Assuming that $\mathbf{p}_{s}(t)=\mathbf{0}$ and $\mathbf{q}_{s}(t)=\mathbf{a} \exp (\overline{\mathrm{i}} \mathrm{s} t)$ we obtain, from Eq. (54), the following nonlinear eigenvalues problem

$$
\left(\mathbf{K}_{s s}+\mathbf{K}_{d d}^{\prime}(\omega)+i \mathbf{K}_{d d}^{\prime \prime}(\omega)-\bar{s}^{2} \mathbf{M}_{s s}\right) \mathbf{a}=\mathbf{0}
$$

where the global matrices $\mathbf{K}_{d d}^{\prime}(\omega)$ and $\mathbf{K}_{d d}^{\prime \prime}(\omega)$ are built from the matrices $\mathbf{K}_{d}^{\prime}(\omega)$ and $\mathbf{K}_{d}^{\prime \prime}(\omega)$ defined in Appendix $\mathrm{C}$ and $\omega$ is the natural frequency of vibration calculated using eigenvalue $S$ and Eq. (39.1).

The iterative procedure was used to solve the eigenvalue problem (55). At the beginning of the iteration process the matrices $\mathbf{K}_{d d}^{\prime}(\omega)$ and $\mathbf{K}_{d d}^{\prime \prime}(\omega)$ are calculated for $\omega=0$ and the resulting linear eigenvalue problem is solved. Next, for the chosen eigenvalues $\bar{s}_{i}$ and the corresponding $\omega_{i}$, the matrices $\mathbf{K}_{d d}^{\prime}(\omega)$ and $\mathbf{K}_{d d}^{\prime \prime}(\omega)$ are recalculated and the next linear eigenvalue problem is solved. The iteration process is considered to have converged when

where $\omega_{i}^{(k)}, \omega_{i}^{(k-1)}, \gamma_{i}^{(k)}$ and $\left|\gamma_{i}^{(k-1)}-\omega_{i}^{(k-1)}\right| \leq \varepsilon \omega_{i}^{(k)},\left|\gamma_{i}^{(k)}-\gamma_{i}^{(k-1)}\right| \leq \varepsilon \gamma_{i}^{(k)}$ damping ratio of interest, respectively, and $\varepsilon$ is the convergence tolerance. 
In this case, the matrix of frequency response functions is defined by

$$
\mathbf{H}(\lambda)=\left[\mathbf{K}_{s s}+\mathbf{K}_{d d}^{\prime}(\lambda)+i \mathbf{K}_{d d}^{\prime \prime}(\lambda)-\lambda^{2} \mathbf{M}_{s s}\right]^{-1}
$$

One important remark can be made concerning the equivalency of the fractional-derivative Kelvin model and the complex modulus model of damper. If $\mathbf{C}_{s s}=\mathbf{0}$ and assuming that all dampers have identical properties and the storage and loss modulus of the fractional-derivative Kelvin model and the complex modulus model are also identical then, taking into account relationships (C.2), (C.9) and (D.6), we can write

$$
\mathbf{K}_{d d}^{\prime}(\omega)=\mathbf{K}_{s s}+\mathbf{C}_{d d} \omega^{\alpha} \cos (\alpha \pi / 2), \quad \mathbf{K}_{d d}^{\prime \prime}(\omega)=\mathbf{C}_{d d} \omega^{\alpha} \sin (\alpha \pi / 2)
$$

Since $i^{\alpha}=\cos (\alpha \pi / 2)+i \sin (\alpha \pi / 2)$, we can conclude that Eqs. (52) and (55) will be identical if $s^{\alpha}=\omega^{\alpha}$. This, however, is not true which means that eigenvalue problems (52) and (55) have different solutions and the considered models of dampers are not identical.

\section{Comparison of dynamic characteristics of structure with VE dampers modelled using different rheological models}

\subsection{Description of a representative structure}

An eight-storey RC frame with three bays and with insufficiently stiff beams is selected for comparison of the influence of the different models of VE dampers on the dynamic characteristics of a structure with dampers. The frame is designed according to the EC8 Part 1 for class B soils. The height of the columns is $3.0 \mathrm{~m}$, the span of the beams is $5.0 \mathrm{~m}$ and Young's modulus for concrete is $31.0 \mathrm{GPa}$. The dimensions of the cross-section of the structural elements are presented in Table 1 while the unit masses of the frame elements are given in Table 2. The data of the structure

Table 1 Dimensions of the eight-storey frame elements

\begin{tabular}{cccc}
\hline \hline Storey level & $\begin{array}{c}\text { Lateral column } \\
{[\mathrm{cm}]}\end{array}$ & $\begin{array}{c}\text { Central column } \\
{[\mathrm{cm}]}\end{array}$ & $\begin{array}{c}\text { Beams } \\
{[\mathrm{cm}]}\end{array}$ \\
\hline 7,8 & $35 \times 35$ & $40 \times 40$ & $30 \times 40$ \\
5,6 & $40 \times 40$ & $45 \times 45$ & $30 \times 45$ \\
3,4 & $45 \times 45$ & $53 \times 53$ & $30 \times 50$ \\
1,2 & $50 \times 50$ & $60 \times 60$ & $30 \times 50$ \\
\hline
\end{tabular}

Table 2 Unit mass of the eight-storey frame elements

\begin{tabular}{cccc}
\hline \hline Storey level & $\begin{array}{c}\text { Unit lateral column } \\
\text { mass } \\
{[\mathrm{kg} / \mathrm{m}]}\end{array}$ & $\begin{array}{c}\text { Unit central column } \\
\text { mass } \\
{[\mathrm{kg} / \mathrm{m}]}\end{array}$ & $\begin{array}{c}\text { Unit beam mass } \\
{[\mathrm{kg} / \mathrm{m}]}\end{array}$ \\
\hline 7,8 & 306.2 & 400.0 & 15000.0 \\
5,6 & 400.0 & 506.2 & 15000.0 \\
3,4 & 506.2 & 702.2 & 15000.0 \\
1,2 & 625.0 & 900.0 & 15000.0 \\
\hline
\end{tabular}


Table 3 Natural frequencies of frame without dampers

\begin{tabular}{cccccc}
\hline \hline \multicolumn{7}{c}{ Natural frequencies [rad/sec] } \\
\hline 3.1311 & 8.6582 & 15.4268 & 23.7804 & 31.2647 & 40.1148 \\
42.1251 & 51.1550 & 52.3598 & 57.6067 & 65.6532 & 69.9862 \\
\hline
\end{tabular}

(except the data concerning the mass per unit length of elements) are taken from the paper by Ribakov and Agranovich (2010).

Table 3 shows the natural frequencies of vibration of the frame without dampers. The dynamic properties of the structure are obtained by two-dimensional analysis of the frame; axial deformations and internal damping of structure are neglected.

\subsection{Parameters of VE damper models}

The dampers are attached on the sixth and seventh floors of the structure. Such location of the dampers is chosen because, in this position, the maximal peak value of the bending moments in the lowest columns is minimal when the structure is subjected to forces produced by the El Centro earthquake. The dampers' location is determined as follows: The equation of motion of structure with one damper connected at the first floor is solved using the Newmark method and the maximal peak value of the bending moments is determined. The calculation is repeated for all the possible positions of the dampers. The position which corresponds to the minimal value of the abovementioned maximal peak value of the bending moments in the columns is chosen as the optimal one. When the position of the first damper is fixed, the same procedure is used to determine the position of the second damper. Of course, the optimal positions of the dampers obtained for one earthquake are not necessarily optimal for other earthquakes. However, optimization of dampers' positions is beyond the scope of our paper. On the other hand, the dampers' positions chosen in this paper are, in some degree, similar to those determined by Agranovich and Ribakov (2010) who found that, on the considered frame, dampers must be connected at the $5^{\text {th }}, 6^{\text {th }}$ and $7^{\text {th }}$ floors.

The dampers are modeled using the following rheological models: i) the complex modulus model, ii) the fractional-derivative Kelvin model; iii) the simple Kelvin model; iv) the simple Maxwell model; v) the generalized Kelvin model with three, five, and seven parameters, and vi) the generalized Maxwell model also with three, five, and seven parameters.

In this paper we do not adopt data from the real experiment. Instead, the storage and loss modulus are calculated from the formulae (D6), which means that the fractional-derivative Kelvin model can be considered as being exact. The chosen parameters of the fractional-derivative Kelvin model are: $\alpha=0.63, k=0.8 \times 10^{6} \mathrm{~N} / \mathrm{m}$ and $c=7.2 \times 10^{6} N \mathrm{sec}^{\alpha} / \mathrm{m}$.

The parameters of the generalized Kelvin model and the generalized Maxwell model, both with seven parameters, are given in Table 4. In Fig. 5 the loss modulus, and the dissipation energy of the above-mentioned models are compared. The dissipation energy is calculated assuming that the amplitude of vibration of damper is equal to $0.01 \mathrm{~m}$ in all of the considered cases. From these figures it can be concluded that the loss modulus and the dissipation energy of the fractionalderivative Kelvin model, the complex modulus model, and both generalized models are almost identical in the considered range of excitation frequency, i.e., in the range $0-15.0 \mathrm{rad} / \mathrm{sec}$. This range of frequency covers the range of the first three natural frequencies of vibration of the considered structure. 
Table 4 Parameters of generalized Kelvin and Maxwell models

\begin{tabular}{cccccc}
\hline \hline & \multicolumn{2}{c}{ Stiffness $\left(\times 10^{6}\right)[\mathrm{N} / \mathrm{m}]$} & & \multicolumn{2}{c}{ Damping factor $\left(\times 10^{6}\right)[\mathrm{N} / \mathrm{m}]$} \\
\hline & Kelvin model & Maxwell model & & Kelvin model & Maxwell model \\
\hline$k_{0}$ & 115.300 & 0.213 & & - & - \\
$k_{1}$ & 36.710 & 66.770 & $c_{1}$ & 5.458 & 2.957 \\
$k_{2}$ & 12.320 & 6.621 & $c_{2}$ & 12.380 & 3.463 \\
$k_{3}$ & 1.109 & 2.886 & $c_{3}$ & 17.350 & 16.610 \\
\hline
\end{tabular}

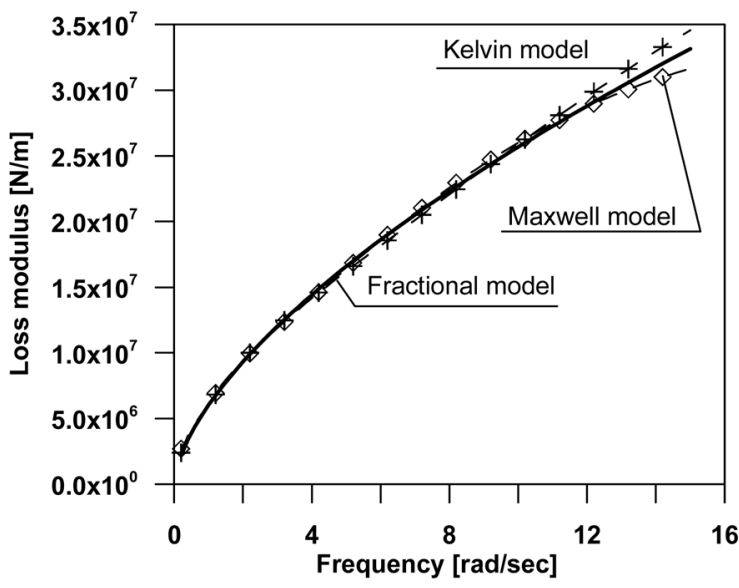

Fig. 5 Comparison of loss modulus

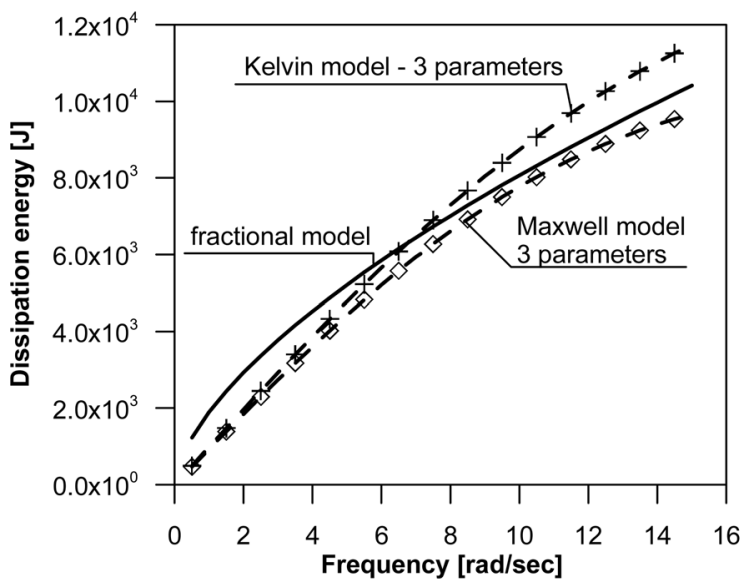

Fig. 7 Comparison of dissipation energy for threeparameter models

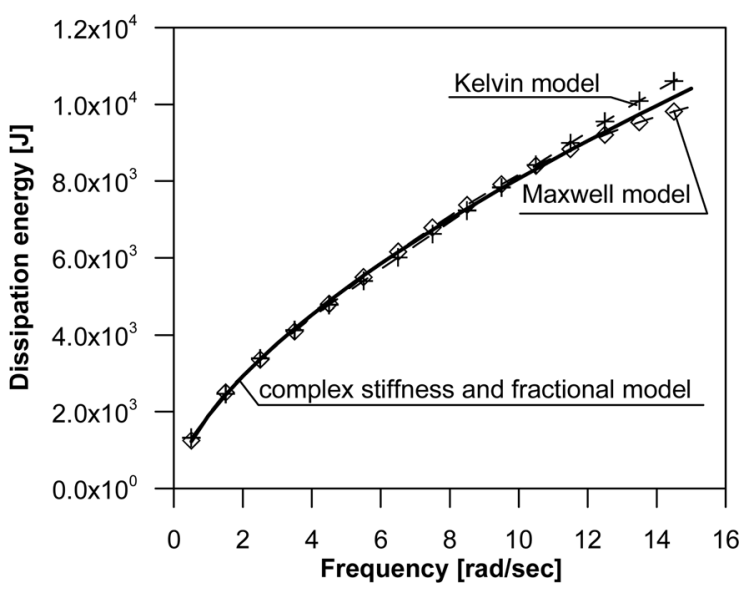

Fig. 6 Comparison of dissipation energy

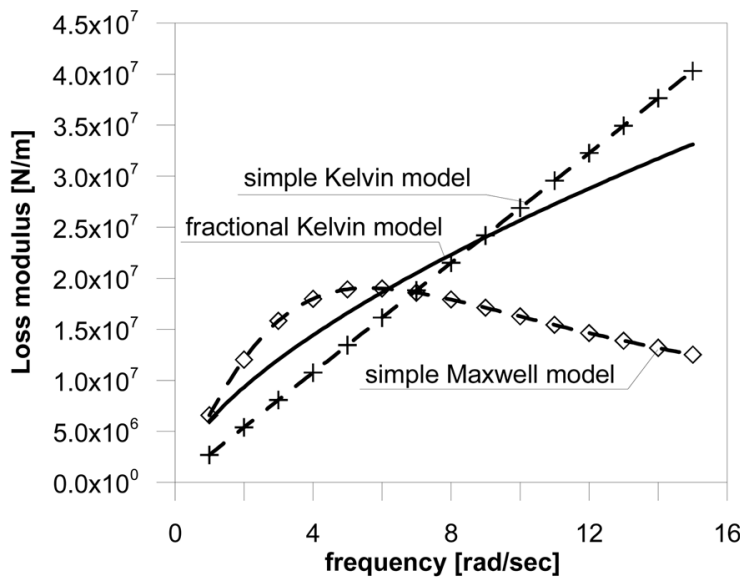

Fig. 8 Comparison of loss modulus for simple models

The values of three and five parameters of the generalized Kelvin and Maxwell models are also given in Table 4. For three-parameter models the $k_{0}, k_{1}$ and $c_{1}$ values are used while $k_{0}, k_{1}, k_{2}, c_{1}$ and $c_{2}$ are the values of the five-parameter models. The comparison of energy dissipated in dampers modeled by the above-mentioned three-parameters models and the fractional-derivative model are shown in Fig. 6. We see that the accuracy of approximation of dissipated energy increases with the 


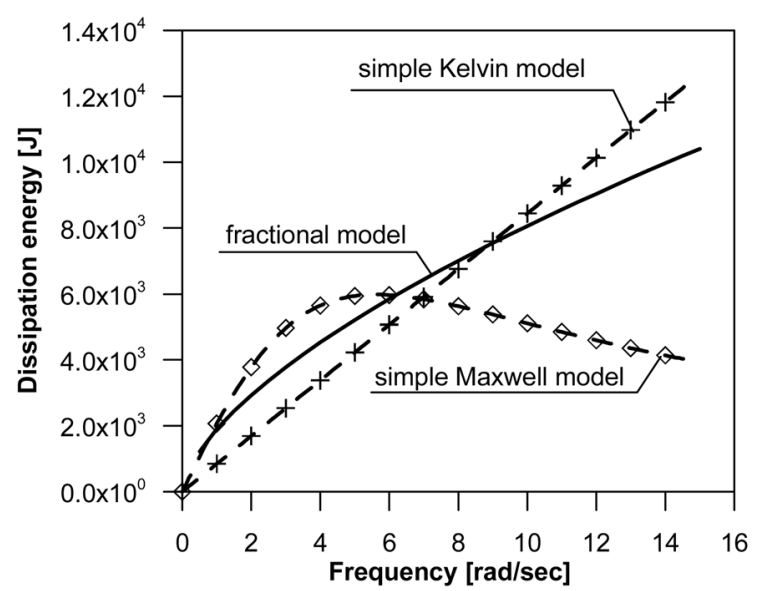

Fig. 9 Comparison of dissipation energy for simple models

number of parameters of generalized models.

The parameters of the simple Kelvin model are: $k=1.49275 \times 10^{7} \mathrm{~N} / \mathrm{m}, c=0.268839 \times 10^{7} \mathrm{~N} / \mathrm{m}$ and the values of parameters of the simple Maxwell model are: $k=3.80783 \times 10^{7} \mathrm{~N} / \mathrm{m}$ and $c=0.677338 \times 10^{7} \mathrm{~N} / \mathrm{m}$. These parameters are calculated by minimizing the mean square norm of difference between the target modules and the analytical modules of the respective model. Comparison between the obtained loss module and the target ones is shown in Fig. 7. Moreover, the dissipation energy of simple models and the dissipation energy of the fractional-derivative model are compared in Fig. 8. As expected, the differences are significant, especially for the simple Maxwell model.

Chevron-braces connect the dampers with the structure. The braces are made of HEB 200 stainless steel profiles of which the parameters are: $E A=1.60105 \times 10^{9} \mathrm{~N}$ and $E J=1.1685 \times 10^{7} \mathrm{Nm}^{2}$.

\subsection{Comparison of dynamic characteristics of considered frame}

Results of the solution to the eigenvalue problems are presented in Tables 5-10. The real and the complex conjugate numbers are obtained as eigenvalues. In Tables 5 and 6 the values of the first three complex conjugate eigenvalues are given for all of the considered models. Results concerning the fractional-derivative Kelvin model and the complex modulus model are given in Table 5.

Table 5First three complex conjugate eigenvalues for a frame with dampers modeled using the fractional derivative Kelvin model and the complex modulus model

\begin{tabular}{ccc}
\hline \hline & Fractional derivative Kelvin model of damper & \\
\hline $1^{\text {st }}$ eigenvalue & $2^{\text {nd }}$ eigenvalue & $3^{\text {rd }}$ eigenvalue \\
\hline$-0.065729 \pm \mathrm{i} 3.20576$ & $-0.85650 \pm \mathrm{i} 9.46271$ & $-0.492826 \pm \mathrm{i} 15.6919$ \\
\hline $1^{\text {st }}$ eigenvalue & Complex modulus model of damper & \\
\hline $3.20630 \pm \mathrm{i} 0.064877$ & $2^{\text {nd }}$ eigenvalue & $3^{\text {rd }}$ eigenvalue \\
\hline
\end{tabular}


Table 6 First three complex conjugate eigenvalues for frame with dampers

\begin{tabular}{cccc}
\hline \hline \multicolumn{4}{c}{ Kelvin model of damper with } \\
\hline 7 parameters & 5 parameters & 3 parameters & 2 parameters \\
\hline$-0.06490 \pm \mathrm{i} 3.20697$ & $-0.05740 \pm \mathrm{i} 3.23237$ & $-0.02938 \pm \mathrm{i} 3.2751$ & $-0.03789 \pm \mathrm{i} 3.22543$ \\
$-0.86607 \pm \mathrm{i} 9.45473$ & $-0.83770 \pm \mathrm{i} 9.67058$ & $-0.66116 \pm \mathrm{i} 10.010$ & $-0.89249 \pm \mathrm{i} 9.35764$ \\
$-0.49557 \pm \mathrm{i} 15.7308$ & $-0.45514 \pm \mathrm{i} 15.8313$ & $-0.35155 \pm \mathrm{i} 15.964$ & $-0.67370 \pm \mathrm{i} 15.6689$ \\
\hline \multicolumn{4}{c}{ Maxwell model of damper with } \\
\hline 7 parameters & 5 parameters & 3 parameters & 2 parameters \\
\hline$-0.06429 \pm \mathrm{i} 3.2066$ & $-0.07035 \pm \mathrm{i} 3.192$ & $-0.06537 \pm \mathrm{i} 3.1553$ & $-0.08317 \pm \mathrm{i} 3.2176$ \\
$-0.84920 \pm \mathrm{i} 9.5529$ & $-0.89831 \pm \mathrm{i} 9.462$ & $-0.97980 \pm \mathrm{i} 9.2092$ & $-0.46152 \pm \mathrm{i} 9.7251$ \\
$-.40766 \pm \mathrm{i} 15.774$ & $-0.42859 \pm \mathrm{i} 15.74$ & $-0.47366 \pm \mathrm{i} 15.660$ & $-0.15489 \pm \mathrm{i} 15.701$ \\
\hline
\end{tabular}

Table 7 Real eigenvalues for frame with dampers

\begin{tabular}{cccc}
\hline \hline \multicolumn{4}{c}{ Kelvin model of damper with } \\
\hline 7 parameters & 5 parameters & 3 parameters & 2 parameters \\
\hline-0.366069 & - & - & -93.46470 \\
-0.378653 & - & - & -120.536 \\
-2.55708 & -2.12886 & - & -30098.8 \\
-2.71947 & -2.27026 & - & -30104.3 \\
-21.2562 & -18.7723 & -15.4856 & - \\
-25.6171 & -22.7386 & -17.4200 & - \\
\hline 7 parameters & Maxwell model of damper with & \\
\hline-0.161840 & 5 parameters & 3 parameters & 2 parameters \\
-0.166646 & - & - & -3.09734 \\
-1.64402 & - & - & -3.67824 \\
-1.74088 & -1.62449 & - & - \\
-12.7486 & -1.73319 & - & - \\
-14.3179 & -12.5245 & -11.8525 & - \\
\hline
\end{tabular}

Table 8 The natural frequencies and non-dimensional damping ratios of frame with dampers - the fractional derivative model and the and complex modulus model

\begin{tabular}{cccc}
\hline \multicolumn{2}{c}{ Natural frequencies $[\mathrm{rad} / \mathrm{sec}]$} & \multicolumn{2}{c}{ Non-dimensional damping ratios } \\
\hline $\begin{array}{c}\text { Fractional } \\
\text { Kelvin model }\end{array}$ & $\begin{array}{c}\text { Complex modulus } \\
\text { model of damper }\end{array}$ & $\begin{array}{c}\text { Fractional } \\
\text { Kelvin model }\end{array}$ & $\begin{array}{c}\text { Complex modulus } \\
\text { model of damper }\end{array}$ \\
\hline 3.20644 & 3.20695 & 0.020499 & 0.020230 \\
9.50139 & 9.52612 & 0.0901444 & 0.0848897 \\
15.6996 & 15.7032 & 0.031391 & 0.0306982 \\
\hline
\end{tabular}


Table 9 The natural frequencies of frame with dampers

\begin{tabular}{|c|c|c|c|c|}
\hline \multirow{2}{*}{$\begin{array}{l}\text { Natural } \\
\text { frequency }\end{array}$} & \multicolumn{4}{|c|}{ Kelvin model of damper with } \\
\hline & $\begin{array}{c}7 \text { parameters } \\
{[\mathrm{rad} / \mathrm{sec}]}\end{array}$ & $\begin{array}{c}5 \text { parameters } \\
{[\mathrm{rad} / \mathrm{sec}]}\end{array}$ & $\begin{array}{c}3 \text { parameters } \\
{[\mathrm{rad} / \mathrm{sec}]}\end{array}$ & $\begin{array}{c}2 \text { parameters } \\
{[\mathrm{rad} / \mathrm{sec}]}\end{array}$ \\
\hline$\omega_{1}$ & 3.20762 & 3.23288 & 3.27528 & 3.22565 \\
\hline$\omega_{2}$ & 9.49431 & 9.70680 & 10.0314 & 9.40010 \\
\hline$\omega_{3}$ & 15.7386 & 15.8378 & 15.9674 & 15.6834 \\
\hline \multirow{2}{*}{$\begin{array}{l}\text { Natural } \\
\text { frequency }\end{array}$} & \multicolumn{4}{|c|}{ Maxwell model of damper with } \\
\hline & $\begin{array}{c}7 \text { parameters } \\
{[\mathrm{rad} / \mathrm{sec}]}\end{array}$ & $\begin{array}{c}5 \text { parameters } \\
{[\mathrm{rad} / \mathrm{sec}]}\end{array}$ & $\begin{array}{c}3 \text { parameters } \\
{[\mathrm{rad} / \mathrm{sec}]}\end{array}$ & $\begin{array}{c}2 \text { parameters } \\
{[\mathrm{rad} / \mathrm{sec}]}\end{array}$ \\
\hline$\omega_{1}$ & 3.20729 & 3.19275 & 3.15601 & 3.21865 \\
\hline$\omega_{2}$ & 9.59056 & 9.50468 & 9.26113 & 9.73603 \\
\hline$\omega_{3}$ & 15.7789 & 15.7483 & 15.6673 & 15.7020 \\
\hline
\end{tabular}

Table 10 Non-dimensional damping ratios of frame with dampers

\begin{tabular}{ccccc}
\hline \hline \multirow{2}{*}{$\begin{array}{c}\text { Damping } \\
\text { ratio }\end{array}$} & \multicolumn{4}{c}{ Kelvin model of damper with } \\
\cline { 2 - 4 } & 7 parameters & 5 parameters & 3 parameters & 2 parameters \\
\hline$\gamma_{1}$ & 0.0202338 & 0.0177562 & 0.0089706 & 0.0117468 \\
$\gamma_{2}$ & 0.0912198 & 0.0863001 & 0.0659092 & 0.0949451 \\
$\gamma_{3}$ & 0.0314874 & 0.0287375 & 0.0220165 & 0.0429562 \\
\hline Damping & & Maxwell model of damper with & \\
ratio & 7 parameters & 5 parameters & 3 parameters & 2 parameters \\
\hline$\gamma_{1}$ & 0.020046 & 0.022035 & 0.020712 & 0.025839 \\
$\gamma_{2}$ & 0.088543 & 0.094512 & 0.105796 & 0.047404 \\
$\gamma_{3}$ & 0.025836 & 0.027215 & 0.030232 & 0.009864 \\
\hline
\end{tabular}

Because the solution to the equation of motion (54) is taken in the form $\mathbf{q}_{s}(t)=\mathbf{a} \exp \left(\mathrm{i}^{-} t\right)$, the real or imaginary parts of $\bar{s}$ must be compared with the imaginary or real part of eigenvalue $s$ of the eigenvalue problem (51), respectively. Results show small differences of which the source is explained at the end of Section 3. The maximal differences reach $5.6 \%$ and $0.3 \%$ for the real and imaginary parts of $s$, respectively.

In Table 6 the first three complex eigenvalues are presented when the classical rheological models of dampers are used. The model with two parameters refers to the simple Kelvin or Maxwell model. After comparing the results obtained for the Kelvin and Maxwell models with seven parameters with the results obtained for the fractional derivative Kelvin model, they are observed to be in good or satisfactory agreement. The maximal differences are $1.3 \%$ and $0.25 \%$ for the real and imaginary part of the Kelvin model with seven parameters, respectively. For the Maxwell model with seven parameters these differences are $17.3 \%$ and $0.95 \%$, respectively. Similar differences are observed when we compare results obtained for the Kelvin or Maxwell model with seven parameters and the results obtained for the complex modulus model.

For the simple Kelvin model discussed, maximal differences are $42.0 \%$ and 1.1\%, respectively. 
Moreover, when dampers are modeled using the simple Maxwell model, the above mentioned differences are $68.0 \%$ and $2.8 \%$ and they are greater than for the simple Kelvin model.

Real eigenvalues are also solutions to the eigenvalue problems when dampers are modeled by the classical rheological models. In the case of the generalized Kelvin and Maxwell models the real eigenvalues reflect the dynamics of internal variables and the number of real eigenvalues is equal to the number of internal variables. Comparing the values of real eigenvalues obtained for both discussed models we see that these values are of the same order but the differences between them are significant. It can be observed that by increasing the number of parameters in the generalized Kelvin or Maxwell model we obtain values which show some convergence trend. If the simple Maxwell model is used to damper modeling then we also obtain the real eigenvalues of which the number is equal to the number of internal variables and which also reflect the dynamics of internal variables.

The real eigenvalues of the eigenvalue problem obtained for the frame with dampers modeled by the simple Kelvin model, which has no internal variables, indicate that two modes of vibration are overdamped. It is the qualitative difference in comparison with the results obtained for other models of dampers. The existence of the discussed real eigenvalues depends on the values of the parameters of the simple Kelvin model.

The first three obtained natural frequencies and non-dimensional damping ratios are presented in Tables 8-10. In terms of these quantities, conclusions concerning differences between the obtained results are similar. Natural frequencies and non-dimensional damping ratios obtained for the frame with dampers modeled using the fractional-derivative Kelvin model and the complex modulus are given in Table 8. Relative differences are not greater than $0.26 \%$ (natural frequencies) or $5.8 \%$ (damping ratios) which means they are in good agreement.

In Table 9 the natural frequencies are given for the frame when dampers are modeled using the simple and generalized Kelvin and Maxwell models. In a similar way, in Table 10, the nondimensional damping ratios are presented. The most accurate results are for the seven-parameter Kelvin and Maxwell models. Relative differences between results obtained for the discussed Kelvin model and the fractional-derivative Kelvin model are not greater than $0.25 \%$ (natural frequencies) and $1.2 \%$ (damping ratios). For the seven-parameter Maxwell model the discussed differences are not greater than $1.0 \%$ (natural frequencies) and $16.8 \%$ (damping ratios). In conclusion we can say that the first three natural frequencies and the non-dimensional damping ratios of the frame show good or satisfactory agreement when VE dampers are modeled by the fractional-derivative model, the complex modulus model or the seven-parameter Kelvin and Maxwell models.

If the number of parameters of the generalized Kelvin model growth then the natural frequencies slightly decrease and the non-dimensional damping ratios increase. Results for the generalized Maxwell model show the opposite trends, i.e. natural frequencies slightly increase and the nondimensional damping ratios decrease with the number of model parameters.

The relative differences of natural frequencies are relatively small (not greater than $5.6 \%$ ) for all of the damper models compared. However, the relative differences of non-dimensional damping ratios could be of the order of $42.0 \%$ and $68.0 \%$ for the simple Kelvin model and the simple Maxwell model, respectively. Consequently, the simple rheological models do not accurately describe the dissipation property of the frame with VE dampers. The three parameter Kelvin model also cannot be recommended as the appropriate one because the discussed differences reach $56.0 \%$. If we accept the relative differences of the order $18.0 \%$ then the remaining classical rheological model could be used to model VE dampers. The relatively large difference of $\gamma_{3}$ (i.e. $17.7 \%$ ) exists 


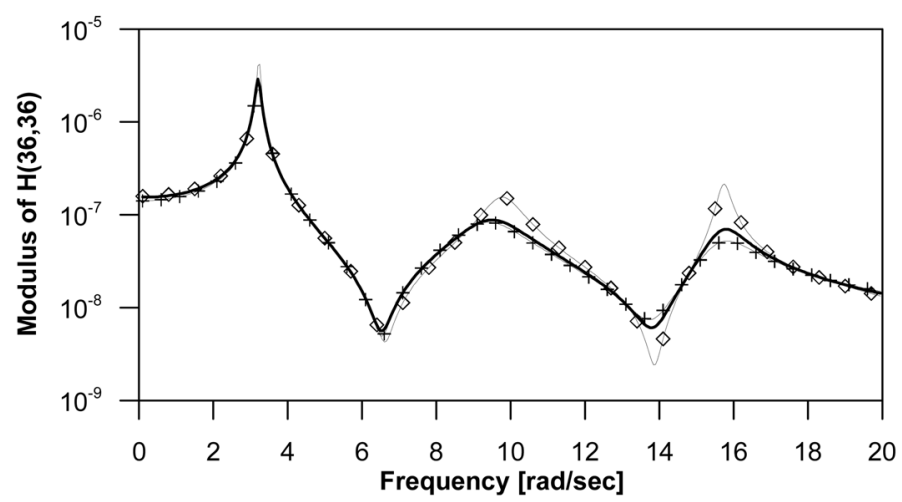

Fig. 10 Modulus of the frequency response function $H_{36,36}(\lambda)$ for fractional derivative Kelvin model (thick line), the simple Kelvin model (thin line with crosses), and for the simple Maxwell model (thin line with rhombs)

for the seven-parameter Maxwell model.

Finally the frequency response functions for the structure with differently modeled VE dampers are compared. Here the element $H_{i j}(\lambda)$ of the matrix frequency response function is interpreted as the displacement of the $i$-th degree of freedom of the structure subjected to the unit harmonically varying force at the $j$-th degree of freedom. The frequency response function $H_{36,36}(\lambda)$ is compared. The displacement number 36 is the horizontal displacement of the eight storeys. The discussed frequency response function calculated for frame with dampers modeled by the fractionalderivative model, the complex modulus model and the seven-parameter Kelvin model are almost identical. The maximal peak differences in the resonances are of the order of $1.0 \%$ for all of the considered resonance areas. These differences are of the order of $20.0 \%$ in the third resonance area when results obtained for the seven-parameter Maxwell model are compared with those obtained for the fractional-derivative Kelvin model.

For the simple Maxwell and Kelvin models, the discussed differences are the greatest. In Fig. 10, comparison of modulus of $H_{36,36}(\lambda)$ obtained for the frame with dampers modeled using the fractional-derivative Kelvin model (the thick line), the simple Kelvin model (the thin line with crosses), and the simple Maxwell model (the thin line with rhombs) are shown. Important differences between the compared frequency functions are visible.

\section{Conlusions}

Several models of dampers, used to describe the dynamic behaviour of frame structures with VE dampers, are considered and compared in this paper. The fractional-derivative Kelvin model, the complex modulus model and a family of rheological models, including the very often used simple Kelvin and Maxwell models, are compared in full detail. The comparison is made in the frequency domain for a carefully chosen frame structure with VE dampers. The formulae for the energy dissipated in a damper modelled in different ways are derived.

Several conclusions can be formulated on the basis of the results of numerical analysis presented above. The most important ones are listed below.

1. Different models are able to correctly describe the dynamic behaviour of VE dampers. In 
particular, the fractional-derivative Kelvin model, the complex modulus mode, and the sevenparameter Kelvin model give us almost identical results. Results suggest that the generalized Maxwell model could also be used if the number of model parameters is sufficient. This conclusion is in agreement with the results presented by Singh and Chang (2009) where the generalized Kelvin and Maxwell models are used as models of VE dampers.

2. The simple Kelvin and the simple Maxwell model are not able to correctly describe, in the frequency domain, the dynamic behaviour of frames with VE dampers. In particular, relative differences concerning the non-dimensional damping ratios are great.

3. In the considered case, the differences between the results obtained for the generalized Maxwell model are greater in comparison with the results obtained using the generalized Kelvin model if both models have an identical number of parameters.

4. The needed number of parameters of the generalized Kelvin and Maxwell models and the fractional-derivative model depends on the frequency range for which the storage and loss modulus of VE dampers must be approximated. Smaller numbers of parameters are needed for the fractionalderivative model.

5. The nonlinear eigenvalue problems must be solved when the complex modulus model or the fractional-derivative model of VE dampers are chosen. The solution procedure for these problems is more complicated than the solution procedure for the linear eigenvalue problems which are obtained for the remaining models.

6. There are some qualitative differences between the results obtained. For the frame with a fixed number of VE dampers the total number of eigenvalues and eigenvectors depends on the chosen model of dampers and the number of parameters of the models. Moreover, for the generalized Kelvin and Maxwell models and the simple Maxwell model both the real and complex numbers are eigenvalues while only the complex numbers are obtained (for sufficiently small damping) when the remaining models are chosen.

7. The dissipative energy is a good measure of equivalence of different models of VE dampers.

\section{Acknowledgments}

The first author wishes to acknowledge the financial support received from the Poznan University of Technology (Grant No. DS 11-068/12) in connection with this work.

\section{References}

Agranovich, G. and Ribakov, Y. (2010), "A method for efficient placement of active dampers in seismicalle excited structures", Struct. Control Hlth. Monit., 17, 513-531.

Barkanov, E., Hufenbach, W. and Kroll, L. (2003), "Transient response analysis of systems with different damping models", Computer Meth. Appl. Mech. Eng., 192, 33-46.

Chang, T.S. and Singh, M.P. (2009), "Mechanical model parameters for viscoelastic dampers", J. Eng. Mech., 135, 581-584.

Chang, T.S. and Singh, M.P. (2002), "Seismic analysis of structures with a fractional derivative model of viscoelastic dampers", Earthq. Eng. Eng. Vib., 1, 251-260.

Christopoulos, C. and Filiatrault, A. (2006), Principles of passive supplemental damping and seismic isolation, IUSS Press, Pavia, Italy.

Connor, J.J. and Klink, B.S.A. (1996), "Introduction to motion-based design", WIT Press. 
Fujita, K., Moustafa, A. and Takewaki, I. (2010) "Optimal placement of viscoelastic dampers and supporting members under variable critical excitations", Earthq. Struct., 1, 43-67.

Hatada, T., Kobori, T., Ishida, M.A. and Niwa, N. (2000), "Dynamic analysis of structures with Maxwell model", Earthq. Eng. Struct. Dyn. Earthq., 29, 159-176.

Lee, S.H., Son, D.I., Kim, J. and Min, K.W. (2004), "Optimal design of viscoelastic dampers using eigenvalue assignment”, Earthq. Eng. Struct. Dyn., 33, 521-542.

Lewandowski, R. and Chorąźyczewski, B. (2010), "Identification of the parameters of the Kelvin-Voigt and the Maxwell fractional models, used to the modeling of viscoelastic dampers", Compos. Struct., 88, 1-17.

Lewandowski, R. and Chorąźyczewski, B. (2007), "Remarks on modelling of passive viscoelastic dampers, Proceedings of the $9^{\text {th }}$ International Conference Modern Building Materials, Structures and Technique, Vilnius, Lithuania, May.

Lewandowski, R. and Pawlak, Z. (2011), "Dynamic analysis of frames with viscoelastic dampers modelled by rheological models with fractional derivatives", J. Sound Vib., 330, 923-936.

Matsagar, V.A. and Jangid, R.S. (2005), "Viscoelastic damper connected to adjacent structures involving seismic isolation", J. Civ. Eng. Mana., 11, 309-322,

Mazza, F. and Vulcano, A. (2007) "Control of the along-wind response of steel framed buildings by using viscoelastic or friction dampers", Wind Struct., 10, 233-247.

Okada, R., Nakata, N., Spencer, B.F., Kasai, K. and Kim, B.S. (2006), "Rational polynomial approximation modeling for analysis of structures with VE dampers", J. Earthq. Eng., 10, 97-125.

Park, J.H., Kim, J. and Min, K.W. (2004), "Optimal design of added viscoelastic dampers and supporting braces", Earthq. Eng. Struct. Dyn., 33, 465-484.

Podlubny, I. (1999), Fractional Differential Equations, Academic Press.

Ribakov, Y. and Agranovich, G. (2011), "A method for design of seismic resistant structures with viscoelastic dampers”, Struct. Des. Tall Spec. Build., 20, 566-578.

Shen, K.L., Soong, T.T., Chang, K.C. and Lai, M.L. (1995), "Seismic behaviour of reinforced concrete frame with added viscoelastic dampers", Eng. Struct., 17, 372-380.

Singh, M.P. and Chang, T.S (2009), "Seismic analysis of structures with viscoelastic dampers", J. Eng. Mech., 135, 571-580.

Singh, M.P. and Moreschi, L.M. (2002), "Optimal placement of dampers for passive response control”, Earthq. Eng. Struct. Dyn., 31, 955-976.

Singh, M.P., Verma, N.P. and Moreschi, L.M. (2003), "Seismic analysis and design with Maxwell dampers", $J$. Eng. Mech., 129, 273-282.

Sorrentino, S. and Fassana, A. (2007), "Finite element analysis of vibrating linear systems with fractional derivative viscoelastic models", J. Sound Vib., 299, 839-853.

Shukla, A.K. and Datta, T.K. (1999), "Optimal use of viscoelastic dampers in building frames for seismic force", J. Struct. Eng., 125, 401-409.

Takewaki, I. (2009) Building control with passive dampers, Optimal performance-based design for earthquakes, Wiley and Sons (Asia), Singapore.

Tsai, M.H. and Chang, K.C. (2002), "Higher-mode effect on the seismic responses of buildings with viscoelastic dampers", Earthq. Eng. Eng. Vib., 1, 119-129.

$\mathrm{Xu}$, Z.D. (2007), "Earthquake mitigation study of viscoelastic dampers for reinforced concrete structures, Vib. Control, 13, 29-43.

Zhang, W.S. and Xu, Y.L. (2000), "Vibration analysis of two buildings linked by Maxwell model defined fluid dampers", J. Sound Vib., 233, 775-796. 


\section{Appendix A. Finite element matrices of the generalized Kelvin model of VE damper}

In this appendix the explicit form of the matrices used to describe the generalized Kelvin model of the VE damper is given.

$$
\begin{aligned}
& \mathbf{K}_{d}=\left[\begin{array}{ll}
\mathbf{K}_{z z} & \mathbf{K}_{z w} \\
\mathbf{K}_{w z} & \mathbf{K}_{w w}
\end{array}\right], \mathbf{C}_{d}=\left[\begin{array}{ll}
\mathbf{C}_{z z} & \mathbf{C}_{z w} \\
\mathbf{C}_{w z} & \mathbf{C}_{w w}
\end{array}\right] \\
& \mathbf{K}_{w w}=\left[\begin{array}{ccccc}
k_{0}+k_{1} & -k_{1} \ldots \ldots 0 & 0 & 0 \ldots \ldots 0 & 0 \\
-k_{1} & k_{1}+k_{2} \ldots 0 & 0 & 0 \ldots \ldots 0 & 0 \\
\ldots \ldots \ldots \ldots & \ldots \ldots \ldots \ldots & \ldots \ldots \ldots \ldots & \ldots \ldots \ldots \ldots & \ldots \ldots \ldots \ldots \\
0 & 0 \ldots \ldots-k_{i-1} & k_{i-1}+k_{1} & -k_{i} \ldots \ldots 0 & 0 \\
\ldots \ldots \ldots . . \ldots \ldots \ldots . . \ldots & \ldots \ldots \ldots \ldots & \ldots \ldots \ldots \ldots & \ldots \ldots \ldots \ldots \\
0 & 0 \ldots \ldots \ldots 0 & 0 & 0 \ldots-k_{m-1} & k_{m-1}+k_{m}
\end{array}\right]
\end{aligned}
$$

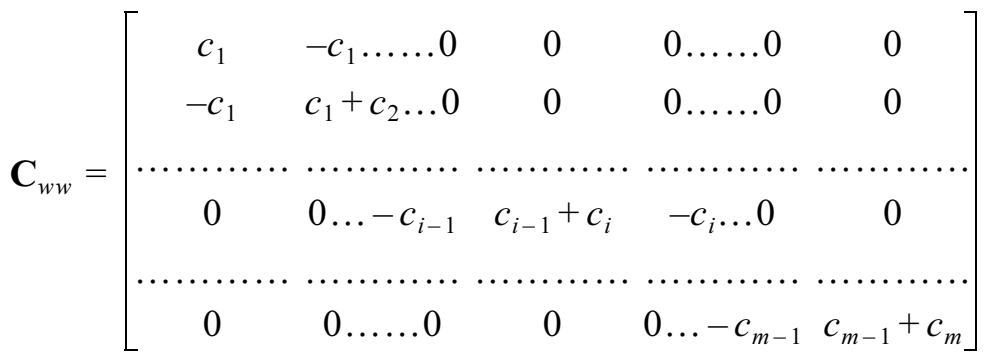

$$
\begin{aligned}
& \mathbf{K}_{z z}=\left[\begin{array}{cccc}
c^{2} k_{0} & c s k_{0} & 0 & 0 \\
c s k_{0} & s^{2} k 0 & 0 & 0 \\
0 & 0 & c^{2} k_{m} & c s k_{m} \\
0 & 0 & c s k_{m} & s^{2} k_{m}
\end{array}\right], \mathbf{C}_{z z}=\left[\begin{array}{cccc}
0 & 0 & 0 & 0 \\
0 & 0 & 0 & 0 \\
0 & 0 & c^{2} c_{m} & c s c_{m} \\
0 & 0 & c s c_{m} & c s c_{m}
\end{array}\right] \\
& \mathbf{K}_{z w}=\mathbf{K}_{w z}^{T}=\left[\begin{array}{cccc}
-c k_{0} & 0 \ldots 0 \ldots & 0 \\
-s k_{0} & 0 \ldots 0 \ldots & 0 \\
0 & 0 \ldots 0 \ldots & -c k_{m} \\
0 & 0 \ldots 0 \ldots & -s k_{m}
\end{array}\right], \mathbf{C}_{z w}=\mathbf{C}_{w z}^{T}=\left[\begin{array}{ccc}
0 & 0 \ldots 0 \ldots & 0 \\
0 & 0 \ldots 0 \ldots & 0 \\
0 & 0 \ldots 0 \ldots & -c c_{m} \\
0 & 0 \ldots 0 \ldots & -s c_{m}
\end{array}\right]
\end{aligned}
$$




\section{Appendix B. Finite element matrices of the generalized Maxwell model of VE damper}

In this appendix the explicit form of matrices used to describe the generalized Maxwell model of the VE damper is given.

$$
\begin{aligned}
& \mathbf{K}=\left[\begin{array}{ll}
\mathbf{K}_{z z} & \mathbf{K}_{z w} \\
\mathbf{K}_{w z} & \mathbf{K}_{w w}
\end{array}\right], \mathbf{C}_{d}=\left[\begin{array}{ll}
\mathbf{C}_{z z} & \mathbf{C}_{z w} \\
\mathbf{C}_{w z} & \mathbf{C}_{w w}
\end{array}\right] \\
& \mathbf{K}_{z w}=\left[\begin{array}{cccc}
-k_{1} & -k_{2} \ldots \ldots & -k_{i} \ldots \ldots & -k_{m} \\
0 & 0 \ldots \ldots & 0 \ldots \ldots & 0 \\
0 & 0 \ldots \ldots & 0 \ldots \ldots & 0 \\
0 & 0 \ldots \ldots & 0 \ldots \ldots & 0
\end{array}\right], \mathbf{C}_{z w}=\left[\begin{array}{cccc}
0 & 0 \ldots \ldots & 0 \ldots & 0 \\
0 & 0 \ldots \ldots & 0 \ldots \ldots & 0 \\
-c_{1} & -c_{2} \ldots & -c_{i} \ldots & -c_{m} \\
0 & 0 \ldots \ldots & 0 \ldots \ldots & 0
\end{array}\right] \\
& \mathbf{K}_{w w}=\operatorname{diag}\left(k_{1}, k_{2}, \ldots \ldots, k_{m}\right), \quad \mathbf{C}_{w w}=\operatorname{diag}\left(c_{1}, c_{2}, \ldots \ldots, c_{m}\right) \\
& {\left[c^{2}\left(k_{0}+\sum_{i=1}^{m} k_{1}\right) c s\left(k_{0}+\sum_{i=1}^{m} k_{i}\right) \quad-c^{2} k_{0} \quad-c s k_{0}\right.} \\
& \mathbf{K}_{z z}=\left[\begin{array}{cccc}
c s\left(k_{0}+\sum_{i=1}^{m} k_{1}\right) s^{2}\left(k_{0}+\sum_{i=1}^{m} k_{i}\right) & -c s k_{0} & -s^{2} k_{0} \\
-c^{2} k_{0} & -c s k_{0} & c^{2} k_{0} & c s k_{0} \\
-c s k_{0} & -s^{2} k_{0} & c s k_{0} & s^{2} k_{0}
\end{array}\right] \\
& \mathbf{C}_{z z}=\left[\begin{array}{cccc}
0 & 0 & 0 & 0 \\
0 & 0 & 0 & 0 \\
0 & 0 & c^{2} \sum_{i=1}^{m} c_{i} c s \sum_{i=1}^{m} c_{i} \\
0 & 0 & c s \sum_{i=1}^{m} c_{i} s^{2} \sum_{i=1}^{m} c_{i}
\end{array}\right], \mathbf{C}_{z w}=\mathbf{C}_{z w}^{T}=\left[\begin{array}{cccc}
0 & 0 \ldots \ldots & 0 \ldots \ldots & 0 \\
0 & 0 \ldots \ldots & 0 \ldots \ldots & 0 \\
-c c_{1} & -c c_{2} \ldots & -c c_{i} \ldots & -c c_{m} \\
-s c_{1} & -s c_{2} \ldots & -s c_{i} \ldots & -s c_{m}
\end{array}\right] \\
& \mathbf{K}_{z w}=\mathbf{K}_{z w}^{T}=\left[\begin{array}{cccc}
-c k_{1} & -c k_{2} \ldots & -c k_{i} \ldots & -c k_{m} \\
-s k_{1} & -s k_{2} \ldots & -s k_{i} \ldots & -s k_{m} \\
0 & 0 \ldots \ldots & 0 \ldots \ldots & 0 \\
0 & 0 \ldots \ldots & 0 \ldots \ldots & 0
\end{array}\right]
\end{aligned}
$$




\section{Appendix C. Finite element matrices of the simple and fractional-derivative Kelvin model, the simple Maxwell model and the complex modulus model of the VE damper}

The explicit form of the vectors and matrices used to describe the simple and fractional-derivative Kelvin model of VE damper is

$$
\begin{aligned}
& \mathbf{q}_{d}(t)=\mathbf{q}_{z}(t)=\operatorname{col}\left(q_{1}(t), q_{2}(t), q_{3}(t), q_{4}(t)\right)
\end{aligned}
$$

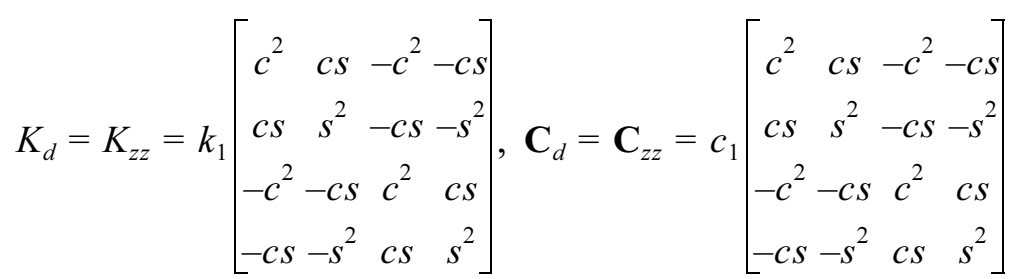

The explicit form of the matrices used to describe the simple Maxwell model of VE damper is

$$
\begin{gathered}
\mathbf{q}_{d}(t)=\operatorname{col}\left(\mathbf{q}_{z}(t), \mathbf{q}_{w}(t)\right), \mathbf{q}_{z}(t)=\operatorname{col}\left(q_{1}(t), q_{2}(t), q_{3}(t), q_{4}(t)\right), \mathbf{q}_{w}(t)=\operatorname{col}\left(q_{w, 1}(t)\right) \\
\mathbf{K}_{d}=\left[\begin{array}{ll}
\mathbf{K}_{z z} & \mathbf{K}_{z w} \\
\mathbf{K}_{w z} & \mathbf{K}_{w w}
\end{array}\right], \mathbf{C}_{d}=\left[\begin{array}{cc}
\mathbf{C}_{z z} & \mathbf{C}_{z w} \\
\mathbf{C}_{w z} & \mathbf{C}_{w w}
\end{array}\right] \\
\mathbf{K}_{z z}=k\left[\begin{array}{llll}
c^{2} & c s & 0 & 0 \\
c s & s^{2} & 0 & 0 \\
0 & 0 & 0 & 0 \\
0 & 0 & 0 & 0
\end{array}\right], \mathbf{K}_{z w}=\mathbf{K}_{z w}^{T}=k_{1}\left[\begin{array}{c}
-c \\
-s \\
0 \\
0
\end{array}\right], \mathbf{K}_{w w}=\left[k_{1}\right] \\
\mathbf{C}_{z z}=c_{1}\left[\begin{array}{llll}
0 & 0 & 0 & 0 \\
0 & 0 & 0 & 0 \\
0 & 0 & c & c s \\
0 & 0 & c s & s^{2}
\end{array}\right], \mathbf{C}_{z w}=\mathbf{C}_{z w}^{T}=c_{1}\left[\begin{array}{c}
0 \\
0 \\
-c \\
-s
\end{array}\right], \mathbf{C}_{w w}=\left[c_{1}\right]
\end{gathered}
$$

The formulae for the energy dissipated in the damper modeled by the simple Kelvin model and for the simple Maxwell models are

$$
\begin{gathered}
E_{d}=\pi \lambda c_{1}\left(a_{3}^{2}+b_{3}^{2}\right) \\
E_{d}=\frac{\pi c_{1} \lambda}{1+\tau^{2} \lambda^{2}}\left(a_{3}^{2}+b_{3}^{2}\right)
\end{gathered}
$$

respectively.

The explicit form of matrices used to describe the complex modulus model of VE damper, in the global coordinate systems is 


$$
K_{d}^{\prime}(\lambda)=K^{\prime}(\lambda)\left[\begin{array}{cccc}
c^{2} & c s & -c^{2} & -c s \\
c s & s^{2} & -c s & -s^{2} \\
-c^{2} & -c s & c^{2} & c s \\
-c s & -s^{2} & c s & s^{2}
\end{array}\right], K_{d}^{\prime \prime}(\lambda)=K^{\prime \prime}(\lambda)\left[\begin{array}{cccc}
c^{2} & c s & -c^{2} & -c s \\
c s & s^{2} & -c s & -s^{2} \\
-c^{2}-c s & c^{2} & c s \\
-c s & -s^{2} & c s & s^{2}
\end{array}\right]
$$

\section{Appendix D. The formulae for the storage modulus and the loss modulus of differ- ent models of VE damper}

The formulae for the storage modulus $K^{\prime}(\lambda)$ and the loss modulus $K^{\prime \prime}(\lambda)$ of the considered rheological models of VE damper are:

i) for the simple Kelvin model

$$
K^{\prime}(\lambda)=k, K^{\prime \prime}(\lambda)=c \lambda
$$

ii) for the simple Maxwell model

$$
K^{\prime}(\lambda)=k \frac{\tau^{2} \lambda^{2}}{1+\tau^{2} \lambda^{2}}, K^{\prime \prime}(\lambda)=k \frac{\tau \lambda}{1+\tau^{2} \lambda^{2}}
$$

iii) for the generalized Kelvin model

$$
K^{\prime}(\lambda)=\frac{L^{\prime}(\lambda)}{L^{\prime 2}(\lambda)+L^{\prime 2}(\lambda)}, K^{\prime \prime}(\lambda)=\frac{L^{\prime \prime}(\lambda)}{L^{\prime 2}(\lambda)+L^{\prime \prime 2}(\lambda)}
$$

where

$$
L^{\prime}(\lambda)=\frac{1}{k_{0}}+\sum_{r=1}^{m} \frac{1}{k_{r}\left(1+\tau_{r}^{2} \lambda^{2}\right)}, L^{\prime \prime}(\lambda)=\sum_{r=1}^{m} \frac{\tau_{r} \lambda}{k_{r}\left(1+\tau_{r}^{2} \lambda^{2}\right)}
$$

iv) for the generalized Maxwell model

$$
K^{\prime}(\lambda)=k_{0}+\sum_{r=1}^{m} k_{1} \frac{\tau_{r}^{2} \lambda^{2}}{1+\tau_{r}^{2} \lambda^{2}}, K^{\prime \prime}(\lambda)=\sum_{r=1}^{m} k_{r} \frac{\tau_{r} \lambda}{1+\tau_{r}^{2} \lambda^{2}}
$$

v) and finally for the fractional-derivative Kelvin Model

$$
K^{\prime}(\lambda)=k+c \lambda^{\alpha} \cos (\alpha \pi / 2), K^{\prime \prime}(\lambda)=c \lambda^{\alpha} \sin (\alpha \pi / 2)
$$

